\title{
Virtual photons in imaginary time: Computing exact Casimir forces via standard numerical-electromagnetism techniques
}

\author{
Alejandro Rodriguez, ${ }^{1}$ Mihai Ibanescu, ${ }^{1}$ Davide Iannuzzi, ${ }^{2}$ J. D. Joannopoulos, ${ }^{1}$ and Steven G. Johnson ${ }^{1}$ \\ ${ }^{1}$ Center for Materials Science and Engineering, Massachusetts Institute of Technology, Cambridge, MA 02139 \\ ${ }^{2}$ Faculty of Sciences, Department of Physics and Astronomy, Vrije Universiteit Amsterdam, The Netherlands
}

\begin{abstract}
We describe a numerical method to compute Casimir forces in arbitrary geometries, for arbitrary dielectric and metallic materials, with arbitrary accuracy (given sufficient computational resources). Our approach, based on well-established integration of the mean stress tensor evaluated via the fluctuation-dissipation theorem, is designed to directly exploit fast methods developed for classical computational electromagnetism, since it only involves repeated evaluation of the Green's function for imaginary frequencies (equivalently, real frequencies in imaginary time). We develop the approach by systematically examining various formulations of Casimir forces from the previous decades and evaluating them according to their suitability for numerical computation. We illustrate our approach with a simple finite-difference frequency-domain implementation, test it for known geometries such as a cylinder and a plate, and apply it to new geometries. In particular, we show that a piston-like geometry of two squares sliding between metal walls, in both two and three dimensions with both perfect and realistic metallic materials, exhibits a surprising non-monotonic "lateral" force from the walls.
\end{abstract}

\section{INTRODUCTION}

One of the most dramatic manifestations of quantum mechanics observed in the last half-century is the Casimir force: a tiny force on an uncharged, source-free body due to changes in the zero-point energy associated with quantum vacuum fluctuations (virtual photons) [1, 2, 3, 4, 5]. There have been many experimental verifications of the Casimir force reported in recent decades [6, 7, 8, but always restricted to simple geometries (parallel plates 9, spheres and plates $[9,10,11,12,13,14,15,16,17,[18$, 19, 20, 21, 22], or crossed cylinders [10, 11, 23]). Moreover, the force in these particular geometries is almost always attractive 24 (except possibly for some unusual material systems [8, 25, 26, 27, 28, 29, 30, 31, 32, 33.) and monotonically decreasing with separation. Thus, one might ask whether it is possible to obtain non-monotonic or even repulsive forces in more complex structures, and more generally whether complex geometries might give rise to unexpected force phenomena. For more complicated geometries, however, calculations become extremely cumbersome and often require drastic approximations, a limitation that has hampered experimental and theoretical work beyond the standard geometries.

In this paper, we explore several ways in which wellestablished, efficient techniques from standard computational electromagnetism can be brought to bear on this problem, in order to predict forces for arbitrary geometries and materials with arbitrary accuracy (no uncontrolled approximations). Starting from the simplest, most direct approaches, we show that practical considerations naturally lead towards a particular method involving the integral of the Minkowski stress tensor by repeated evaluation of the imaginary-frequency Green's function - a method previously developed for purely analytical calculations [3, 34, 35. We illustrate the method by a simple finite-difference implementation, but evaluation of the Green's function is so standard that many more sophisticated techniques are immediately applicable, and we discuss what techniques are likely to be optimal. Our approach is tested for geometries with known solutions, and then is applied to new geometries in two and three dimensions that lead to surprising nonmonotonic effects. We also demonstrate the application of our technique to dispersive dielectric materials, not just for idealized perfect metals. We explain how the same technique can be used for visualization of the Casimir interactions between bodies, as well as for computing other quantities of interest, such as torques. The key advantage of exploiting standard computational approaches is not merely that existing code, error analyses, and other experience can be applied to the Casimir problem, but also that these methods have been proven to scale to large three-dimensional problems, which have previously seemed out of reach of exact methods for the Casimir force.

The most common approach to predicting the Casimir force has been to consider approximations for small perturbations around known solutions, such as parallel plates or dilute gases. For parallel plates in $d$ dimensions, separated by a distance $a$, there is a well-known attractive force that scales as $1 / a^{d+1}$, first predicted by Casimir [1] and later extended to formulas for any planarmultilayer dielectric distribution $\varepsilon(x, \omega)$ via the generalized Lifshitz formula 36. A direct, intuitive extension of this result is the proximity force approximation (PFA) 37, which treats the force between two surfaces as a two-body interaction given by the sum of "parallel plate" contributions. Valid in the limit of small curvature, PFA provides an easy way to conceptualize the Casimir force in complex geometries as a simple two-body force law, but unfortunately it may also be deceptive: outside its range of applicability, the Casimir force is not additive 22 and may be qualitatively different from PFA's predictions [38, 39, 40, 41, 42, 43, 44. Other perturbative approaches include renormalized Casimir- 
Polder [45, 46] or semi-classical interactions [47, multiple scattering expansions [48, 49, classical ray optics approximations [50], higher-order PFA corrections [51, and other perturbative techniques 52. The ray-optics approach is especially interesting because, although it is only strictly valid in the small-curvature limit, it captures multiple-body interactions and can therefore sometimes predict the qualitative behavior in cases where other approximations fail [53, 54, 55. Nevertheless, none of these methods provide any guarantees of accuracy in arbitrary geometries, where they involve uncontrolled approximations. Therefore, for complex new geometries, where one might hope to encounter behaviors very different from those in the parallel-plate limit, a different approach is required.

To this end, researchers have sought "exact" numerical methods applicable to arbitrary geometries - that is, methods that converge to the exact result with arbitrary accuracy given sufficient computational resources. One such method was proposed by Ref. 56, based on a path-integral representation for the effective action; this method has predicted the force between a cylinder and a plate [57, and between corrugated surfaces [40, 41]. It is based on a surface parameterization of the fields coupled via vacuum Green's functions, requiring $O\left(N^{2}\right)$ storage and $O\left(N^{3}\right)$ time for $N$ degrees of freedom, making scaling to three dimensions problematic. Another exact method is the "world-line approach" [58, based on Monte-Carlo path-integral calculations. The scaling of the world-line method involves a statistical analysis, determined by the relative feature sizes in the geometry, and is discussed below. The methods of Ref. 56 and Ref. 58 have currently only been demonstrated for perfect-metallic $z$-invariant structures - in this case, the vector unknowns can be decomposed into TE $(\mathbf{E} \cdot \hat{\mathbf{z}}=0)$ and $\operatorname{TM}(\mathbf{H} \cdot \hat{\mathbf{z}}=0)$ scalar fields with Neumann and Dirichlet boundary conditions, respectively - although generalizations have been proposed [58, 59]. Here, we propose a method based on evaluation of the mean Minkowski stress tensor via the fluctuation-dissipation theorem, which only involves repeated evaluation of the electromagnetic imaginary-frequency Green's function. For our initial volume discretization with $N$ degrees of freedom and an efficient iterative solver, this requires $O(N)$ storage and at best $O\left(N^{2-1 / d}\right)$ time in $d$ dimensions. Furthermore, because evaluation of the Green's function is such a standard problem in classical computational electromagnetism, it will be possible to exploit many developments in fast solvers, based on finiteelement 60, 61, 62, 63, spectral 60, 64, or boundaryelement methods 60, 61, 62, 65, 66, 67. As we argue below, a future implementation using boundary-element methods should attain nearly $O(N \log N)$ time. To illustrate the method, however, our initial implementation is based on the much simpler finite-difference frequencydomain method [68] with a conjugate-gradient solver [69], as described below.

In the following sections, we describe the step-by-step conceptual development of our computational method. Our purpose here is to start back at the beginning, with the earliest theoretical descriptions of the Casimir force, and analyze these formulations from the point of view of their suitability for purely numerical calculations. Although the final technique, in terms of the stress tensor integrated over space and imaginary frequency, can be viewed as a numerical implementation of a textbook result due to Dzyaloshinskiî et al. [3, 35, 70, it is illustrative to derive it as the culmination of a sequence of simpler approaches, in order to show how it circumvents a number of numerical obstacles that would hinder a more direct method. To begin with, we illustrate the methods using the well-known case of parallel plates where they can be compared to analytical expressions, but a more rigorous test is subsequently provided by the situation of a cylinder and plate, recently solved numerically [57]. Finally, we apply our method to a new geometry of a "piston"-like structure involving blocks sliding between parallel walls, in both two and three dimensions with both perfect metals and more realistic dispersive dielectrics, and demonstrate a surprising non-monotonic "lateral" effect from the walls. We conclude by analyzing the scaling of the method compared to previous approaches and discussing the application of more sophisticated finite-element and boundary-element techniques.

\section{A SIMPLISTIC APPROACH}

Perhaps the simplest approach to computing the Casimir force is to think of it as the derivative of the zero-point energy $U$ expressed as a sum of ground-state photon energies $\hbar \omega / 2$. For each photon mode with frequency $\omega$, the zero-point energy is $\hbar \omega / 2$, and thus the total Casimir energy, at least for non-dissipative systems where $\omega$ is real [36], is formally given by the sum over all modes [1, 2]:

$$
U=\sum_{\omega} \frac{1}{2} \hbar \omega
$$

This sum is formally infinite, because the classical harmonic modes $\omega$ have unbounded frequencies. There is some controversy over the physical interpretation of this divergence [71, but in practice it is removed by regularizing the sum in some fashion, for example multiplying by $e^{-s \omega}$ for $s>0$, and taking $s \rightarrow 0$ only after the sum is differentiated to obtain the force $F=-d U / d a$ between two bodies with separation $a$ [72. This approach, which was an early method to analytically compute the force between perfect-metal plates [1, might seem to provide the most direct computational method as well. After all, the computation of electromagnetic eigenmodes is routine even in very complicated geometries, and efficient methods are known [60, 61, 62, 73. Unfortunately, it turns out not to be practical for this problem (except in one-dimensional geometries [74]), as explained below, 
but the reason why it is impractical points the way to more efficient methods.

To illustrate the difficulty in directly evaluating Eq. (1), let us consider the simplest one-dimensional geometry: two parallel perfect-metal plates, separated by a distance $a$, in which case one can predict analytically an attractive force $F=\pi \hbar c / 24 a^{2}$ [71]. Ignoring this analytical result, let us apply a numerical method that, conceptually, we could apply to an arbitrary geometry:

1. Discretize space with resolution $\Delta x$ using a finitedifference approximation, with space truncated to a finite computational cell (e.g. with periodic boundaries).

2. Solve numerically for the eigenmode frequencies $\omega$ and sum to obtain $U(a)$.

3. Shift one body (one plate) by one pixel $\Delta x$ and thus compute $U(a+\Delta x)$.

4. Obtain the force $F \approx-[U(a+\Delta x)-U(a)] / \Delta x$.

Note that this method automatically provides its own regularization: the number of modes $\omega$ in a discretized computational cell is finite (the frequencies are bounded by the Nyquist limit), and hence $U$ is finite for $\Delta x>$ 0 . The periodic boundaries will lead to artificial "wraparound" forces, but since Casimir forces decay rapidly with distance, the contribution of these forces can be made negligible for a sufficiently large computational cell.

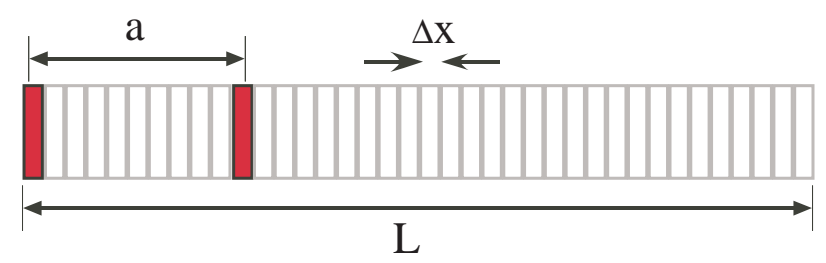

FIG. 1: (Color) Schematic of one dimensional geometry, showing two $1 \mathrm{~d}$ metal plates separated by a distance $a$, embedded in a computational cell of length $L=a+4 a$, with periodic boundary conditions, and resolution $\Delta x$.

This method, for the one-dimensional parallel-plate geometry, is illustrated in Fig. 1. Here, we have two plates with separation $a$ and an overall computational cell size $L=5 a$ (which will contribute an erroneous wrap-around force $1 / 16$ of the physical force). Maxwell's equations, in one dimension, can be written as the scalar eigenproblem $\nabla^{2} E_{z}=\omega^{2} E_{z}$ (in $c=1$ units), which is discretized to

$$
\frac{E_{n+1}-2 E_{n}+E_{n-1}}{\Delta x^{2}}=\omega_{n}^{2} E_{n}
$$

in a center-difference approximation for $E_{z}(n \Delta x)=E_{n}$. For two metal plates with separation $d$ and Dirichlet boundary condition $E_{z}=0$, the discrete eigenvalues $\omega_{n}$ can be found analytically:

$$
\omega_{n}=\frac{2}{\Delta x} \sin \left(\frac{n \pi \Delta x}{2 d}\right),
$$

for $n=0, \ldots, d / \Delta x$. The energy $U$ is then given by summing $\omega_{n}$ in Eq. (1) for $d=a$ and $d=L-a$, and the force $F$ by the discrete derivative of $U$ as above.

Applying this procedure numerically for $\Delta x=0.05 a$, one obtains the correct force to within 5\% (and to any desired accuracy by increasing $L$ and decreasing $\Delta x$ ), so at first glance it may seem that the method is successful. However, its impracticality is revealed if we examine the contribution of each frequency $\omega_{n}$ to the force.
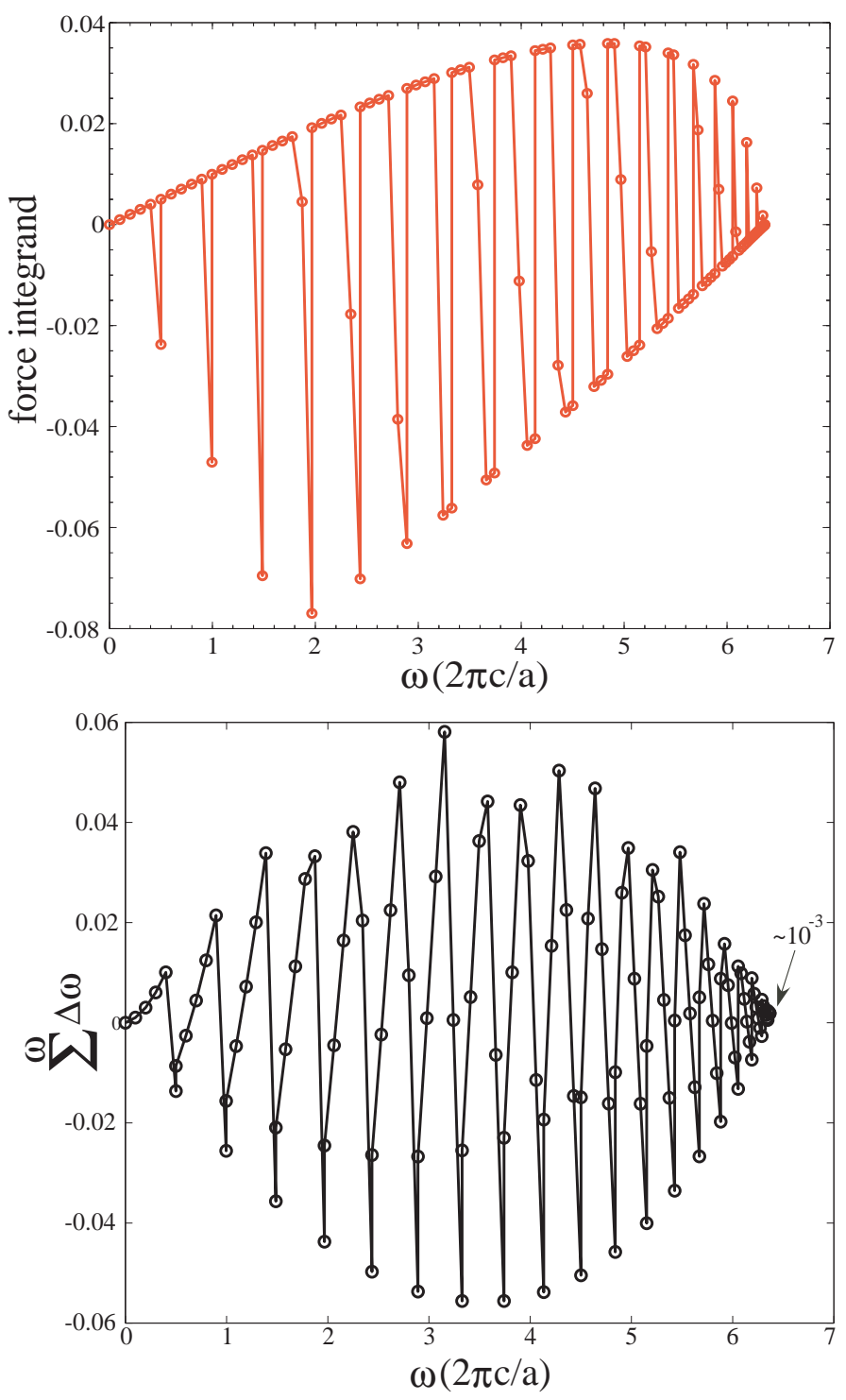

FIG. 2: (Color) top: Plot of force summand, or spectral density, $\hbar \Delta \omega / 2 \Delta x$ vs. $\omega$ for $1 \mathrm{~d}$ parallel metal plates from Fig. 1 . bottom: Plot of force partial sum $\sum^{\omega} \hbar \Delta \omega / 2 \Delta x$ vs. $\omega$.

The top panel of Fig. 2 shows the contribution 
$\hbar \Delta \omega / 2 \Delta x[\Delta \omega=\omega(a+\Delta x)-\omega(a)]$ to the force summation, and the bottom panel shows the corresponding partial sum (for frequencies up to $\omega$ ). We see that every frequency (of the regularized/finite-resolution problem) makes a non-negligible contribution to the force, and the summation is of a wildly oscillating quantity that leaves a tiny remainder at the end. The reason for these oscillations is quite simple: as $a$ is increased, the frequencies on the $4 a$ side of the plates increase slowly, while the smaller number of frequencies on the $a$ side of the plate decrease more rapidly, and these lead to the positive and negative contributions in the top panel of Fig. 2, respectively.

These two features, which are intrinsic properties not limited to this particular discretized geometry [75, combine to make this method impractical in higherdimensional structures. Because every frequency contributes to the force, in a numerical method one must compute every eigenvalue of the Maxwell eigenproblem. In one dimension, that is not so bad, but in general if there are $N$ degrees of freedom ( $N$ grid points), then computing every eigenvalue of an $N \times N$ matrix requires $O\left(N^{2}\right)$ storage and $O\left(N^{3}\right)$ time. This is impractical in three dimensions where $N$ may be in the millions. Furthermore, the wild oscillations of the summand imply that the eigenvalues must be computed quite accurately, and may exacerbate numerical difficulties in larger problems.

However, these undesirable features are avoidable, because we have not yet exploited a key property of Maxwell's equations: causality. If we ignore the causality constraint, then the oscillatory spectrum would be an observable effect: one would simply employ a material that is a perfect metal in some frequency range and transparent otherwise, in order to obtain the force spectrum integrated only in that range. Such a material, however, would violate the Kramers-Kronig constraints that follow from causality considerations [76]. Thus, we are motivated to exploit causality in some fashion to avoid the oscillatory spectrum.

\section{WICK ROTATION AND ENERGY DENSITY}

In order to exploit causality, we will rewrite Eq. (1) in terms of the electromagnetic Green's function via an integral over the density of states. Causality implies that the Green's function has no poles in the upper-half plane, so one can perform a contour integration, or Wick rotation, to transform the sum over real frequencies into an integral along the imaginary-frequency axis. The result of this standard trick turns out to be a well-known expression: an integral of the mean electromagnetic energy density, evaluated by the fluctuation-dissipation theorem using the temperature (Matsubara) Green's function. Again, we focus on the method's suitability as a purely numer$i c a l$ approach, for arbitrary geometries, and we will find that it still leaves something to be desired.

First, we can express the zero-point energy of Eq. (1) as an integral over the local density of states $D(\mathbf{x}, \omega)$ :

$$
U=\frac{\hbar}{2} \int_{0}^{\infty} d \omega \int \omega D(\mathbf{x}, \omega) d^{3} \mathbf{x} .
$$

Since we are solving for the eigenstates of Maxwell's equations $\left(\nabla \times \nabla \times-\omega^{2} \varepsilon\right) \mathbf{E}=0$, the local density of states $D(\mathbf{x}, \omega)$ can be expressed in terms of the Green's tensor $G_{j k}[77]$ :

$$
D(\mathbf{x}, \omega)=\frac{1}{\pi} \frac{d\left(\omega^{2} \varepsilon\right)}{d \omega} \sum_{k=1}^{3} \operatorname{Im}\left\langle\mathbf{x} ; \hat{\mathbf{e}}_{\mathbf{k}}\left|\frac{1}{\nabla \times \nabla \times-\omega^{2} \varepsilon+i 0^{+}}\right| \mathbf{x} ; \hat{\mathbf{e}}_{\mathbf{k}}\right\rangle=\frac{1}{\pi} \frac{d\left(\omega^{2} \varepsilon\right)}{d \omega} \operatorname{Im} \operatorname{tr} G(\omega ; \mathbf{x}-\mathbf{x})
$$

where $G_{j k}$ solves: $\left(\nabla \times \nabla \times-\omega^{2} \varepsilon\right) \mathbf{G}_{k}\left(\omega ; \mathbf{x}-\mathbf{x}^{\prime}\right)=$ $\delta^{3}\left(\mathbf{x}-\mathbf{x}^{\prime}\right) \hat{\mathbf{e}}_{k}$, with $\hat{\mathbf{e}}_{k}$ denoting the unit vector in the $k$ th direction. For non-dissipative systems in which Eq. (1) is valid, $\varepsilon$ is real and we can therefore pull the Im outside of the integral. (The generalization to dissipative materials is discussed below.)

Furthermore, we know from causality requirements that the Green's function has no poles in the upper half plane in $\omega$-space [2, 76]. This means that one can perform a contour integration to relate $\int_{0}^{\infty} d \omega$ to the integral $\int_{0}^{\infty} d w$ along the imaginary-frequency axis $\omega=i w$, also known as a Wick rotation. We therefore obtain:

$$
U=\frac{\hbar}{2 \pi} \int_{0}^{\infty} d w \int w \frac{d\left[w^{2} \varepsilon(i w)\right]}{d w} \operatorname{tr} G(i w ; \mathbf{x}-\mathbf{x}) d^{3} \mathbf{x},
$$

where the new problem to be solved is that of finding the solutions to the imaginary-time Green's function $(c=1$ units):

$$
\left[\nabla \times \nabla \times+w^{2} \varepsilon(i w, \mathbf{x})\right] \mathbf{G}_{k}\left(i w ; \mathbf{x}-\mathbf{x}^{\prime}\right)=\delta^{3}\left(\mathbf{x}-\mathbf{x}^{\prime}\right) \hat{\mathbf{e}}_{k} .
$$

As usual, this is formally infinite, because the Green's function is singular at $\mathbf{x}=\mathbf{x}^{\prime}$, but one typically regularizes the problem by subtracting the vacuum Green's function, which removes the singularity without changing 
the net force.

Equation (6) is not a new result, nor is it limited to non-dissipative materials (unlike our derivation) [3, 36. In fact, it is equivalent to the mean energy in the fluctuating electromagnetic field, derived from the fluctuationdissipation theorem via the temperature Green's functions [3]. Our purpose in deriving it this way is to emphasize the connection to the simplistic approach of Eq. (1). In particular, the mean energy in the electromagnetic fields (for the case of non-magnetic materials $\mu=1$ ) is given by $[3,76$ :

$$
\begin{aligned}
U & =\int_{0}^{\infty} d w \int \frac{1}{2}\left[\frac{d(w \varepsilon)}{d w}\left\langle\mathbf{E}^{2}\right\rangle_{w}+\left\langle\mathbf{H}^{2}\right\rangle_{w}\right] d^{3} \mathbf{x} \\
& =\int_{0}^{\infty} d w \int \frac{1}{2 w} \frac{d\left(w^{2} \varepsilon\right)}{d w}\left\langle\mathbf{E}^{2}\right\rangle_{w} d^{3} \mathbf{x}
\end{aligned}
$$

using the fact that $\int \varepsilon\left\langle\mathbf{E}^{2}\right\rangle_{w}=\int\left\langle\mathbf{H}^{2}\right\rangle_{w}$. Here, the key point is that the mean values of the fluctuating fields are given, via the fluctuation-dissipation theorem, in terms of the imaginary-frequency Green's function [3]:

$$
\left\langle E_{j}(\mathbf{x}) E_{k}\left(\mathbf{x}^{\prime}\right)\right\rangle_{w}=\frac{\hbar}{\pi} w^{2} G_{j k}\left(i w ; \mathbf{x}-\mathbf{x}^{\prime}\right) .
$$

where the dyadic Green's function $G_{i j}$ solves Eq. (7) and obeys the usual boundary conditions on the electric field from classical electromagnetism [76. Substituting Eq. (9) into Eq. (8), one recovers Eq. (6).

From a computational perspective, the imaginaryfrequency integral of Eq. (6) turns out to be greatly superior to the real-frequency summation of Eq. (1), for two reasons. First, while every real frequency $\omega$ contributed to the force $-d U / d a$, the same is not true for the derivative of the imaginary-frequency integrand. In particular, as discussed below, the force integrand in imaginary frequencies turns out to be a smooth, nonoscillatory, strongly peaked function of $w$, meaning that one can integrate it via a smooth-quadrature method that evaluates the integrand at only a small number of $w$ values. Second, the imaginary-frequency Green's function turns out to be quite easy to obtain by relatively standard methods, including for dissipative systems (where obtaining the eigenmodes is harder because it involves a non-Hermitian eigenproblem). These two favorable features are closely related.

Consider Eq. (7) for the imaginary-frequency Green's function. Unlike its real-frequency counterpart, the linear operator on the left-hand side of this equation is real-symmetric and positive-definite (for $w>0$ ). This is true even for dissipative materials where $\varepsilon(\omega)$ is complex, since causality requirements imply that $\varepsilon(i w)$ is purely real and positive (in the absence of gain) 22, 76]. For one thing, this implies that the most powerful numerical methods are applicable to solving the linear system of Eq. (7) - many of these methods (e.g. the conjugategradient method) are restricted to Hermitian positivedefinite operators 69. Also, the resulting Green's func- tion is particularly well-behaved: it is exponentially decaying and non-oscillatory. This transforms the highly oscillatory real- $\omega$ force integrand into a mostly nonoscillatory integrand, and also makes the force integrand exponentially decaying for large $w$ (for large $w$, the interactions between bodies become exponentially small). (In addition, as we will discuss in Sec.VIII] the exponentially decaying Green's function is especially favorable for boundary-element numerical methods.)

Again, considering the simplest possible finitedifference scheme, this leads us to the following numerical algorithm to compute the force:

\section{For a given imaginary frequency $w$ :}

(a) For every grid point $\mathbf{x}$, solve Eq. (77) for each polarization $k$ to obtain $G_{k k}(i w ; \mathbf{x}-\mathbf{x})$.

(b) Sum over $\mathbf{x}$ to compute the spatial integral in Eq. (6).

(c) Repeat the above for the body shifted by one pixel $\Delta x$ and subtract to obtain the force integrand at $w$.

2. Employ a smooth quadrature scheme to integrate the above function over $w$ to obtain the force.

Again, the spatial discretization provides its own regularization $\left(G_{k k}\right.$ is finite), and thus no additional regularization is required (the contribution of the vacuum Green's function to the net force is zero). Again, one can truncate the computational cell in a number of ways, for example with periodic boundaries, and the artifacts thereby introduced will decrease rapidly with cell size. Again, there are also many other ways that one could potentially solve for $G_{k k}$ besides a finite-difference approximation, but we will delay discussion of those techniques until we have formulated the final method in the next section.

The above procedure can again be applied to the onedimensional problem of the force between two plates, as in Fig. 2, to illustrate its basic features. The result is shown in Fig. 3, plotting the force integrand as a function of imaginary frequency $w$, and the difference from Fig. 2 is striking. The integrated force is the same (correct) result as before. Unlike Fig. 2, the force integrand has no sign oscillations, is exponentially decaying for large $w$, and is strongly peaked around a characteristic $w=2 \pi c / a$, corresponding to a "wavelength" of $a$ (the separation). These features imply that the force can be accurately integrated by an adaptive Gauss-Kronrod quadrature scheme [78] using at most a few dozen $w$ points.

Although this method is much more efficient than the one described in the previous section, and is potentially practical at least in two dimensions, it still has some undesirable features. Suppose that we have $N$ grid points in our discretized operator ( $N$ may be very large in 3d). Even if we have an ideal iterative solver for the sparse linear system of Eq. (7), such as an ideal multigrid solver [63, 79, each evaluation of the Green's function 


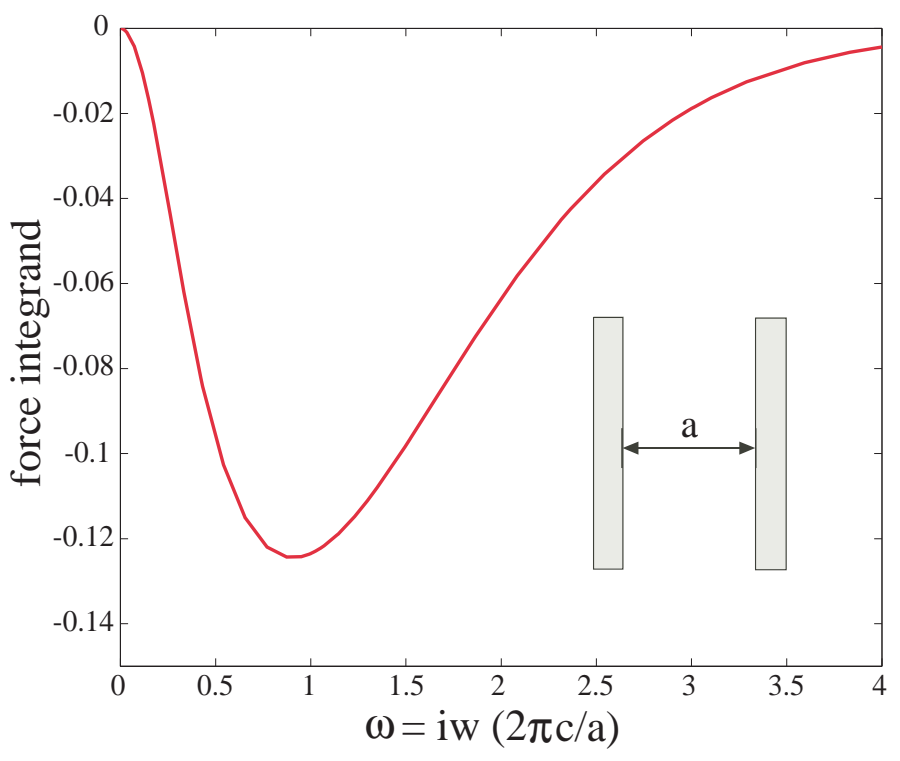

FIG. 3: (Color) Plot of Casimir force integrand $d U / d a$ between two $1 \mathrm{~d}$ parallel plates separated by a distance $a=1$ versus imaginary frequency $w=\operatorname{Im} \omega$, using the method of Sec. III

takes at best $O(N)$ time with $O(N)$ storage. However, we must evaluate the Green's function $3 N$ times in order to perform the spatial integration, resulting in $O\left(N^{2}\right)$ complexity. As is discussed in the next section, one can do much better than this by using the stress tensor instead of the energy density. In fact, as is discussed in Sec. VIII, it should ultimately be possible to obtain the force with nearly $O(N \log N)$ work using a boundaryelement method to compute the stress tensor, in which $N$ is only the number of degrees of freedom required to represent the interfaces between materials.

\section{STRESS-TENSOR COMPUTATIONAL APPROACH}

After having analyzed the feasibility of several techniques to solve the Casimir problem through the lens of numerical electromagnetism, we are ready to appreciate and explore the most feasible of the methods thus far presented: an approach based on the Maxwell stress tensor.

As derived by Dzyaloshinskil et al. 3, 35, 70, the net Casimir force on a body can be expressed as an integral over any closed surface around the body of the mean electromagnetic stress tensor $\left\langle T_{i j}\right\rangle$, integrated over all frequencies. Again, using the same arguments as above, it is computationally convenient to perform a Wick rotation, expressing the net force as an integral over imaginary frequencies $\omega=i w$. (The original derivation used imaginary frequencies to start with, via the temperature Green's function, but the result is equivalent to a Wick rotation of the real-frequency expression. A related analytical treatment, but using purely real $\omega$ and therefore unsuitable for numerical computation because of the oscillations discussed above, has also been examined [80, 81].) The resulting net force is:

$$
\mathbf{F}=\int_{0}^{\infty} d w \oiiint_{\text {surface }}\langle\mathbf{T}(\mathbf{r}, i w)\rangle \cdot d \mathbf{S} .
$$

In two or one dimensions, one or two of the spatial integrals are omitted, respectively, but the result still has the units of force; the change in dimensions of $d \mathbf{S}$ is balanced by a change in the dimensions of the delta function in Eq. (7). On the other hand, for a 3d structure that is $z$-invariant [a constant $2 \mathrm{~d}$ cross-section $\varepsilon(x, y)$ ] or $y z$-invariant [a univariate $\varepsilon(x)$ ], the integrals over the invariant directions are replaced by integrals over the corresponding wavevectors, resulting in a net force per unit length or per unit area, respectively. (These wavevector integrals are discussed in more detail in Sec. VI]) The stress tensor is defined as usual by:

$$
\left\langle T_{i j}(\mathbf{r}, i w)\right\rangle=\mu(\mathbf{r}, i w)\left[\left\langle H_{i}(\mathbf{r}) H_{j}(\mathbf{r})\right\rangle-\frac{1}{2} \delta_{i j} \sum_{k}\left\langle H_{k}(\mathbf{r}) H_{k}(\mathbf{r})\right\rangle\right]+\varepsilon(\mathbf{r}, i w)\left[\left\langle E_{i}(\mathbf{r}) E_{j}(\mathbf{r})\right\rangle-\frac{1}{2} \delta_{i j} \sum_{k}\left\langle E_{k}(\mathbf{r}) E_{k}(\mathbf{r})\right\rangle\right]
$$

where $\mu$ and $\varepsilon$ are the relative permeability and permittivity, respectively, although in most cases we set $\mu=1$ for simplicity (since most materials have negligible magnetic response at short wavelengths, and in any case the stress tensor is normally evaluated over a surface lying in vacuum). As before, the connection to quantum mechanics arises from the correlation functions of the fluctuating fields, given via the fluctuation-dissipation theorem in 
terms of the imaginary- $\omega$ dyadic Green's function. The correlation function for the electric field $\left\langle E_{i} E_{j}\right\rangle$ is given in Eq. (9). In this case, however, we also need the magneticfield correlation functions, which can be obtained by differentiating the electric-field Green's function [3]:

$$
\left\langle H_{i}(\mathbf{r}) H_{j}\left(\mathbf{r}^{\prime}\right)\right\rangle=-\frac{\hbar}{\pi}(\nabla \times)_{i \ell}\left(\nabla^{\prime} \times\right)_{j m} G_{\ell m}\left(i w ; \mathbf{r}-\mathbf{r}^{\prime}\right),
$$

(Alternatively, the $\left\langle H_{i} H_{j}\right\rangle$ correlation function can be computed from the magnetic Green's function, which is the magnetic field in response to a given magnetic-dipole current.) The above expressions are given at zero temperature; the nonzero-temperature force is found by changing $\int d w$ in Eq. 10 into a discrete summation [3, 49]. Although the Green's function (and thus $\mathbf{T}$ ) is formally infinite at $\mathbf{r}=\mathbf{r}^{\prime}$, this divergence is conventionally removed by subtracting the vacuum Green's function; in a numerical method with discretized space, as below, there is no divergence and no additional regularization is required. (The vacuum Green's function gives zero net contribution to the $d \mathbf{S}$ integral, and therefore need not be removed as long as the integrand is finite.)

Historically, this stress-tensor expression was used to derive the standard Lifshitz formula for parallel plates, where $G_{i j}$ is known analytically. Its adaptability and suitability as a purely computational method does not seem to have been recognized, however. As in the previous section, the method involves computing the Green's function for many imaginary frequencies $w$ and spatial points $\mathbf{x}$, integrated over $w$ and $\mathbf{x}$. However, a quick glance at Eq. 10 will suggest at least two obvious computational advantages compared to the method discussed in Sec. III. First, in framing the problem in terms of the stress tensor, we have reduced the spatial integral over the whole volume (Eq. 8) to a surface integral around the body of interest. This implies that, for a $d$-dimensional geometry, the computational effort due to spatial integration is reduced from $O\left(N^{2}\right)$ to $O\left(N^{2-1 / d}\right)$. Second, the force is now given directly in terms of the dyadic Green's function (via the stress tensor), rather than its derivative, which avoids another layer of computation. Moreover, although our derivation is only valid when the stress tensor is evaluated at points within lossless dielectrics (regardless of whether the bodies themselves are dissipative), one can also extend it for evaluation in absorbing media 70. However, the case discussed above (bodies separated by vacuum) is the most common.

So far, we have presented the step-by-step development of an efficient approach to computing Casimir forces. In what follows, we illustrate our new approach using a proof-of-concept finite-difference implementation, and present results that demonstrate its flexibility and utility.

\section{THE FINITE-DIFFERENCE METHOD}

At this point, all that remains is the numerical computation of the Green's function via Eq. (7) for an imaginary frequency $\omega=i w$. This is simply the inversion of a linear operator $\left[\nabla \times \nabla \times+w^{2} \varepsilon(\mathbf{r}, i w)\right]$ that has the convenient properties of being real-symmetric and positivedefinite, as stated above. Almost any technique developed for computational electromagnetism is applicable here, modified to operate at an imaginary frequency. To illustrate our approach, we used a very simple, yet extremely general, method: finite-difference frequencydomain (FDFD) discretization of Eq. (7) in a staggered Yee grid [68], which we then invert by a conjugategradient method 69]. Although the Yee grid in principle allows second-order-accurate finite-difference approximations, unfortunately the whole scheme becomes only first-order-accurate once a discontinuous dielectric function $\varepsilon$ is included. (There are ways to treat interfaces more accurately 82, but we did not implement them here.) Moreover, a very high resolution is often required to resolve the stress tensor close to a dielectric boundary due to the Green's function divergences as a boundary is approached [65]. Despite its shortcomings, however, we found FDFD to be sufficient to obtain accurate results (to within a few percent in a reasonable time) for twodimensional, and three-dimensional $z$-invariant, geometries. The key advantage of FDFD is its flexibility: with very little effort, we were able to implement support for arbitrary geometric shapes and arbitrary materials (both perfect metals and dispersive/dissipative dielectrics).

Again choosing the simplest possible approach, we apply periodic boundary conditions at the edges of the computational cell, which are accurate as long as the boundaries are sufficiently far compared to the separation between the interacting bodies. That is, the periodicity leads to artificial "wrap-around" forces that decay rapidly with cell size $L$ (at least as $1 / L^{3}$ in $2 \mathrm{~d}$ ); we chose cell sizes large enough to make these contributions negligible $(<1 \%)$.
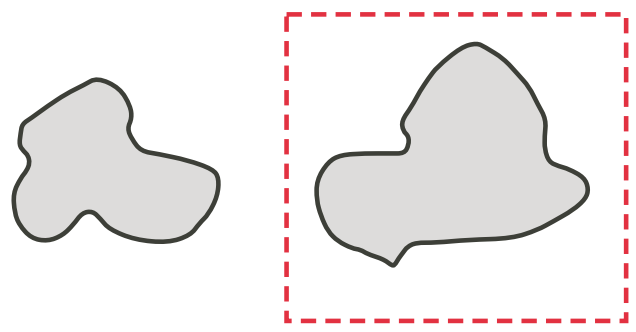

FIG. 4: (Color) Schematic illustration of a possible contour around a body; the force on the body is given by an integral of the stress tensor around this contour.

The computational process (using a simple finitedifference scheme) goes as follows:

1. Pick a contour/surface around the body of interest, as in Fig. 4 (which will typically not coincide with 
the boundary of the body).

2. For a given frequency $w$ :

(a) For every grid point $\mathbf{x}$ on the discretized contour/surface, solve Eq. (7) for each polarization $k$ to obtain $G_{j k}(i w ; \mathbf{x}-\mathbf{x})$.

(b) Integrate the resulting stress tensor $T_{j k}$ over the surface, as in Eq. 10.

3. Integrate the above function over $w$ to obtain the force; since the integrand is a smooth function of $w$, an efficient adaptive quadrature scheme can be employed [78.

Although this scheme does not require any additional regularization (the integrand is finite for a finite resolution, and the integral of the vacuum stress tensor over the contour is zero), we have found that numerical convergence can be accelerated by subtracting the stresstensor integral over the isolated bodies. For example, in the schematic of Fig. 4, we would first compute the stress-tensor integral for the two bodies as shown, then subtract the integral of the stress tensor over the same surface with one body removed, and then subtract again for the stress tensor with the other body removed. Of course, these subtracted quantities are zero in the limit of infinite spatial resolution - there is no net force on an isolated body. However, at a finite resolution the discretization error at the interface between two materials can lead to a finite force that vanishes as resolution is increased. By subtracting this error term from the force, we find that the numerical error is typically reduced by an order of magnitude or so. We emphasize, however, that this is merely an optimization - even without subtraction, the force converges to the correct result, and merely requires a somewhat higher resolution.

\section{FORCES IN TRANSLATION-INVARIANT STRUCTURES}

It is common to solve for the Casimir force between bodies that are translation-invariant in one or more directions; for example, between a cylinder and a plate [57. that are invariant in the $z$ direction. More generally, one might consider structures that are periodic in some direction with a non-zero period $\Lambda$, where $\Lambda \rightarrow 0$ corresponds to translation invariance. Intuitively, in such cases one need only perform computations in the unit cell of the periodicity, and the spatial integration in the invariant direction(s) is replaced by integration over a wavevector k from Bloch's theorem [83, 84. Although special cases of this familiar idea are well known in Casimir computations [56, 84, here we provide a review of this approach for an arbitrary periodicity in the context of stress-tensor computational methods; the detailed derivation is provided in the Appendix. We also mention a useful optimization for the common special case of perfect-metallic $z$-invariant structures.
Let us consider a single direction of periodicity: suppose that the structure is periodic in $z$ with period $\Lambda$. In this case, it is natural to choose a surface for our stresstensor integral that is also periodic in $z$. For example, imagine that Fig. 4 depicts a two-dimensional $(x y)$ crosssection of a $z$-invariant structure, and the dashed line depicts a cross-section of the corresponding $z$-invariant stress-tensor surface. Because the total force is infinite, the quantity of interest is the force per unit $z$. It is convenient to consider the net force $\mathbf{F}$ from a finite length $L=N \Lambda$ with periodic boundaries, and obtain the force per unit length as $\lim _{N \rightarrow \infty} \mathbf{F} / L$. Naively, $\mathbf{F} / L$ can be written directly via Eq. 10$)$, where we break the integral over $z$ into a summation over the unit cells:

$$
\begin{aligned}
\frac{\mathbf{F}}{L} & =\frac{1}{L} \int_{0}^{\infty} d w \sum_{n=0}^{N-1} \iint \mathbf{T}(i w ; \mathbf{r}-n \Lambda \hat{\mathbf{z}}) \cdot d \mathbf{S} \\
& =\frac{1}{\Lambda} \int_{0}^{\infty} d w \iint \mathbf{T}(i w ; \mathbf{r}) \cdot d \mathbf{S}
\end{aligned}
$$

where the surface integral is over the portion of the surface lying in the unit cell only, and in the second line we have used the fact that the stress tensor is periodic. This expression is inconvenient, however, because the direct evaluation of $\mathbf{T}(i w, \mathbf{r})$ requires the response to a single point source in the large- $L$ structure, and a single point source does not produce a periodic field (requiring a full three-dimensional calculation even for a $z$-invariant structure). Rather, one would like to consider the field in response to periodic point sources, which produce a periodic field that can be treated by a small computational cell with periodic boundary conditions. This is accomplished by Fourier-transforming the expressions in Eq. (13) and taking the $N \rightarrow \infty$ limit, as described in detail by the Appendix. The resulting force per unit length is:

$$
\frac{\mathbf{F}}{L}=\frac{1}{\Lambda} \int_{0}^{\infty} d w \int_{-\pi / \Lambda}^{\pi / \Lambda} \frac{d k_{z}}{2 \pi} \iint \mathbf{T}\left(i w, k_{z} ; \mathbf{r}\right) \cdot d \mathbf{S},
$$

where the surface integral is still over the portion of the surface lying in the unit cell. Here, $\mathbf{T}\left(i w, k_{z} ; \mathbf{r}\right)$ denotes the stress tensor computed from the Green's functions for Bloch-periodic boundaries - that is, from the fields in response to a periodic set of point-dipole sources with phase $e^{i k_{z} \Lambda n}$ in the $n$th unit cell. This stress tensor can be computed using a computational cell that is only one unit cell in the $z$ direction, e.g. by a two-dimensional computational cell for a $z$-invariant structure. [Equivalently, $\mathbf{T}\left(i w, k_{z} ; \mathbf{r}\right)$ could instead be computed from the Green's function for ordinary periodic boundaries, but with $\nabla$ replaced by $\nabla+i k_{z} \hat{\mathbf{z}}$ [83.] Just as for $w$, the stress tensor is a smooth function of $k_{z}$ and therefore the $k_{z}$ integral can be computed by an efficient quadrature scheme (e.g. Gaussian quadrature).

If the structure is periodic (or invariant) in more than one direction, one simply repeats the above procedure: 
for each periodic direction, we only consider the portion of the stress-tensor integral in the unit cell, with Bloch-periodic boundary conditions, and integrate over the corresponding Bloch wavevector component. Also, by symmetry one only need integrate over the irreducible Brillouin zone of the structure [83, e.g. in one dimension (where time-reversal symmetry normally equates $k_{z}$ and $\left.-k_{z}\right)$ the $\int_{-\pi / \Lambda}^{\pi / \Lambda} d k_{z}$ integral can be replaced by $2 \int_{0}^{\pi / \Lambda} d k_{z}$.

For the common case of a $z$-invariant perfect-metal structure (i.e. one has a homogeneous $\varepsilon$ surrounded by perfect metal), there are several important simplifications. First, the $\left(k_{z}, w\right)$ Green's function is exactly the same as the $\left(0, \sqrt{k_{z}^{2}+w^{2}}\right)$ solution [57]. Therefore, we need only compute the $k_{z}=0$ solutions at each $w$, and weight the $d w$ integrand by a factor of $\pi w$ (the circumference of a semi-circle of radius $w$ ) divided by the $2 \pi$ that would appear in the $d k_{z}$ integral. (For two directions of translational symmetry, one would weight the integral by the area of a hemisphere of radius $w$, and in general, in $d$ dimensions, by the area of a radius- $w$ hypersphere.) Furthermore, at $k_{z}=0$, the solutions can be divided into two scalar polarizations, TE $(\mathbf{E} \cdot \hat{\mathbf{z}}=0)$ and $\mathrm{TM}(\mathbf{H} \cdot \hat{\mathbf{z}}=0)[76]$.

\section{NUMERICAL RESULTS AND VISUALIZATION}

In the following sections we demonstrate our method's validity by checking it against known results for perfect metals, and in particular for the case of a cylinder adjacent to a plate. A new geometry that displays an interesting non-monotonic behavior is presented for both perfect and realistic dispersive metals, and in both $2 \mathrm{~d}$ and 3d. Furthermore, we explain how one can use stresstensor maps to visualize the interactions between bodies and identify the most important spatial regions.

\section{A. Parallel plates in one dimension}

First, for comparison to the one-dimensional parallelplate integrands plotted in Fig. 2 and Fig. 3, we plot the corresponding integrand for the stress tensor in Fig. 5 Again, this integrates to the correct force $\left(\pi \hbar c / 24 a^{2}\right.$ for each polarization), but we note that the integrand is not identical to the integrand from the imaginary-frequency energy derivative.

Since the one-dimensional parallel-plate force is commonly derived from the Lifshitz formula, which in turn is derived from the stress tensor, this cannot be regarded as a rigorous validation of our method (except in the most basic sense of checks for bugs in our code).

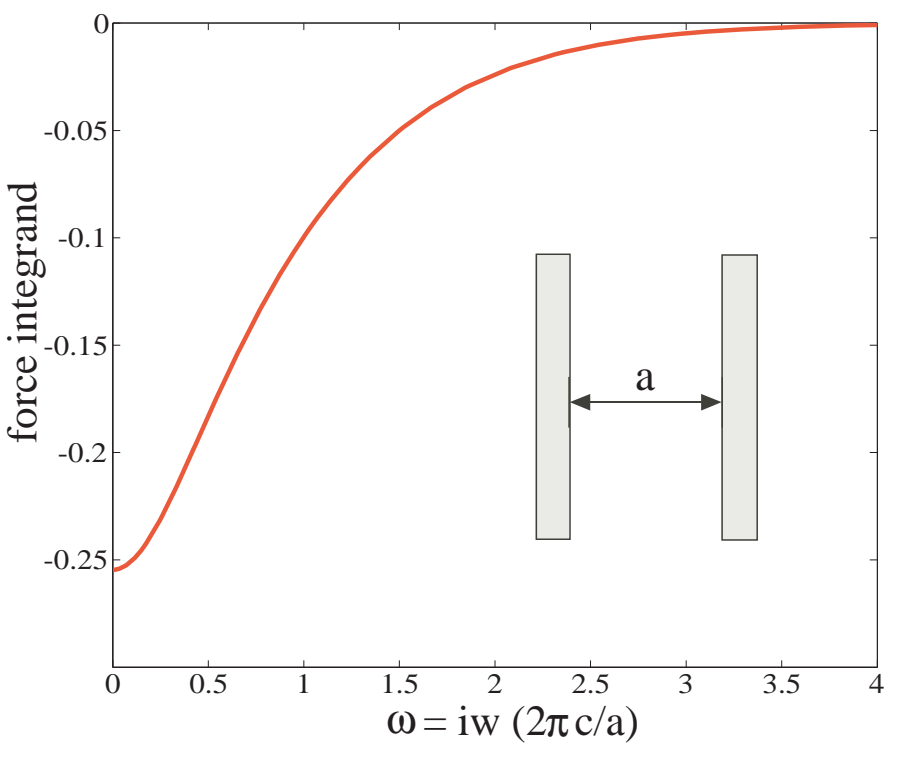

FIG. 5: (Color) Plot of Casimir force integrand $\oiint T \cdot d \mathbf{S}$ between two $1 \mathrm{~d}$ parallel plates separated by a distance $a=1$ versus imaginary frequency $w=\operatorname{Im} \omega$, using the stress-tensor method of Sec. IV]

\section{B. Cylinder and plate}

A more complicated geometry, consisting of a perfect metallic cylinder adjacent to a perfect metallic plate in three dimensions, was solved numerically by Ref. [57, to which our results are compared in Fig. 6. Ref. 57 used a specialized Fourier-Bessel basis specific to this cylindrical geometry, which should have exponential (spectral) convergence. Our use of a simple uniform grid was necessarily much less efficient, especially with the firstorder accuracy, but was able to match the Ref. 57] results within $\sim 3 \%$ using reasonable computational resources. A simple grid has the advantage of being very general, as illustrated below, but other general bases with much greater efficiency are possible using finite-element or boundary-element methods; the latter, in particular, could use a spectral Fourier basis similar to Ref. 57 and exploit a fast-multipole method or similar $O(N \log N)$ solver technique. Surface discretizations (boundary elements) will also have the advantage that the infinite amount of space surrounding the objects is treated analytically rather than having to be truncated with some boundary conditions (here, periodic). This is discussed in greater detail in Sec. VIII

Also shown, in the inset of Fig. 6, is a plot of the interaction stress-tensor component $\Delta\left\langle T_{x x}\right\rangle$ at a typical imaginary frequency $w=2 \pi c / a$. By "interaction" stress tensor $\Delta\left\langle T_{i j}\right\rangle$, we mean the total $\left\langle T_{i j}\right\rangle$ of the full geometry minus the sum of the $\left\langle T_{i j}\right\rangle$ 's computed for each body in isolation. Here, the stress tensors of the isolated cylinder and plate have been subtracted, giving us a way to visualize the force due to the interaction. As described further below, such stress plots reveal the spa- 


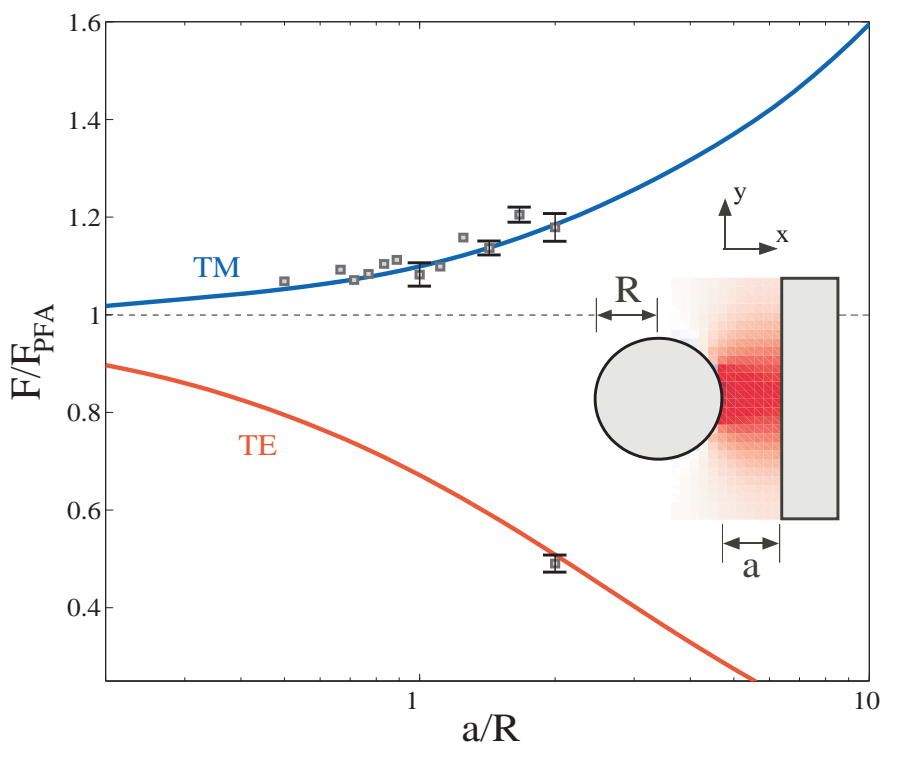

FIG. 6: (Color) Casimir force between a $3 \mathrm{~d}$ radius- $R$ cylinder and a plate (inset), relative to the proximity-force approximation $F_{\mathrm{PFA}}$, vs. normalized separation $a / R$. The solid lines are the Casimir force computed in Ref. 57 for TE (gray) and TM (blue) polarizations, along with results computed by our method with a simple finite-difference discretization (gray squares). Error bars were estimated for some data points by using computations at multiple spatial resolutions. Inset shows interaction stress tensor $\Delta\left\langle T_{x x}\right\rangle$ at a typical imaginary frequency $w=2 \pi c / a$, where red indicates attractive stress.

tial regions in which two bodies most strongly affect one another, and therefore reveal where a change of the geometry would have the most impact. (In contrast, Ref. 85 plots an interaction-energy density that does not directly reveal the force, since the force requires the energy to be differentiated with respect to $a$. For example, Ref. 85] s subtracted energy density apparently goes nearly to zero as a metallic surface is approached, whereas the stress tensor cannot since the stress integration surface is arbitrary.)

\section{Two-dimensional metal piston and non-monotonic "lateral" forces}

We now consider a more complicated geometry in which there are interactions between multiple bodies: a two-dimensional "piston"-like structure, shown in Fig. 7. consisting of two metal $s \times s$ squares separated by a distance $a$ from one another (here, $s=a$ ) and separated by a distance $h$ from infinite metal plates on either side. We then compute the Casimir force between the two squares, in two dimensions (that is, for $z$-invariant fields, unlike the cylinder case above where $z$ oscillations were included), as a function of the separation $h$. The result for perfect conductors is shown in Fig. 7. plotted for the TE and TM polarizations and also showing the total

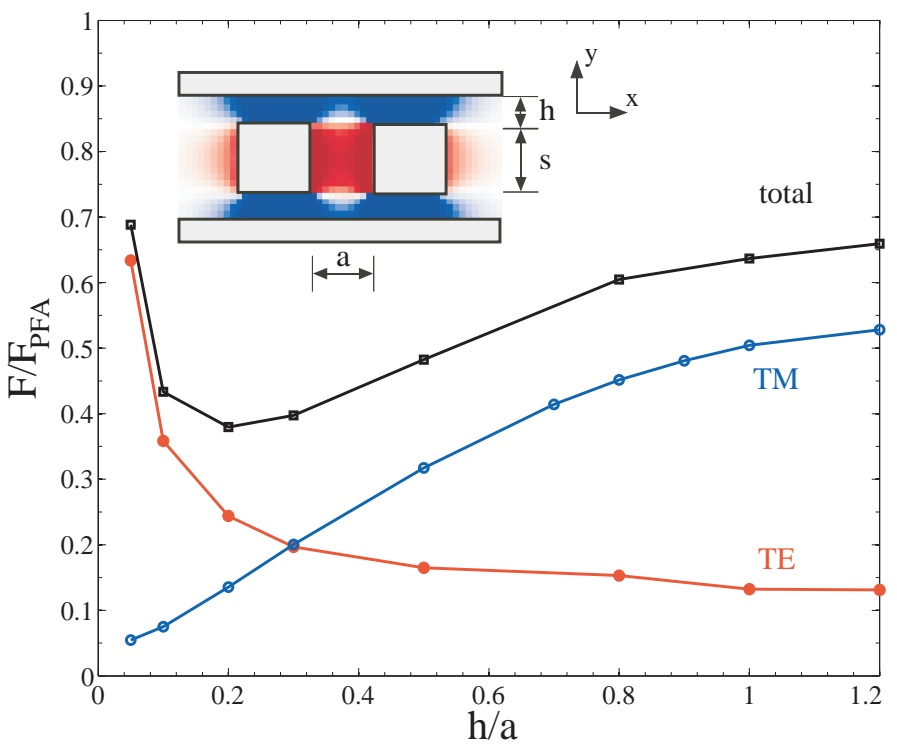

FIG. 7: (Color) Casimir force between 2d (z-invariant fields) metal squares $F / F_{\mathrm{PFA}}$, vs. distance from metal plate $h$ (inset), normalized by the total force (TE+TM) obtained using the PFA, $F_{\mathrm{PFA}}=\hbar c \zeta(3) s / 8 \pi a^{3}$. The total force is plotted (black squares) along with the TE (red dots) and TM (blue circles) contributions.

force. (Error bars are not shown because the estimated error is $<1 \%$. This structure is computationally easier than the cylinder-plate problem, for a finite-difference discretization, because the metallic walls allow the computational cell size to be small in at least one direction.) In the limit of $h \rightarrow 0$, this structure approaches the $2 \mathrm{~d}$ "Casimir piston," which has been solved analytically for the TM polarization [86]. Our results, extrapolated to $h=0$, agree agree with this analytical result to within $3 \%$ (although we have computational difficulties for small $h$ due to the high resolution required to resolve a small feature in FDFD). For $h>0$, however, the result is surprising in at least two ways. First, the total force is non-monotonic in $h$, due to a competition between the TE and TM contributions to the forces. Second, the $h$ dependence of the force is a lateral effect of the parallel plates on the squares, which would be zero by symmetry in PFA or any other two-body-interaction approximation.

The reader may notice that the TE and TM forces in the cylinder-plate case, Fig. 6, also have opposite-sign slopes in the graph, and one may therefore suspect that non-monotonic forces could occur in that case as well. However, in the cylinder-plate case this apparent difference in sign is merely an artifact of the normalization: the PFA force varies with the separation $a$, and when the actual force (which is monotonically decaying with $a$ ) is divided by this variable force one can obtain "nonmonotonic" plots. (A similar "non-monotonic" plot can be seen in Ref. [57.) In contrast, for Fig. 77 the PFA normalization is constant because $a$ is fixed, and thus the 
relative forces from different $h$ are directly comparable.

Although lateral forces can still arise qualitatively in various approximations, such as in ray optics or in PFA restricted to "line-of-sight" interactions, it may not be immediately clear how these could predict nonmonotonicity. We also note that, in the large- $h$ limit, the force remains different from PFA due to finite-s "edge" effects [85], which are captured by our method. It turns out that one can qualitatively predict the non-monotonic behavior, due to the competition between TE and TM forces, using the ray-optics approximation, although this approximation is not quantitatively accurate except for $h=0$; we will describe this ray-optics analysis in a future publication [55]. (a)

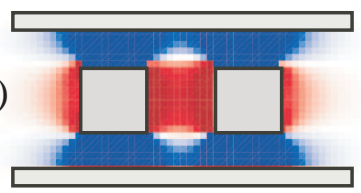

(d)

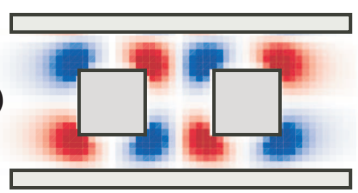

(b)

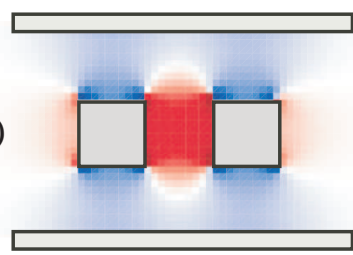

(c)
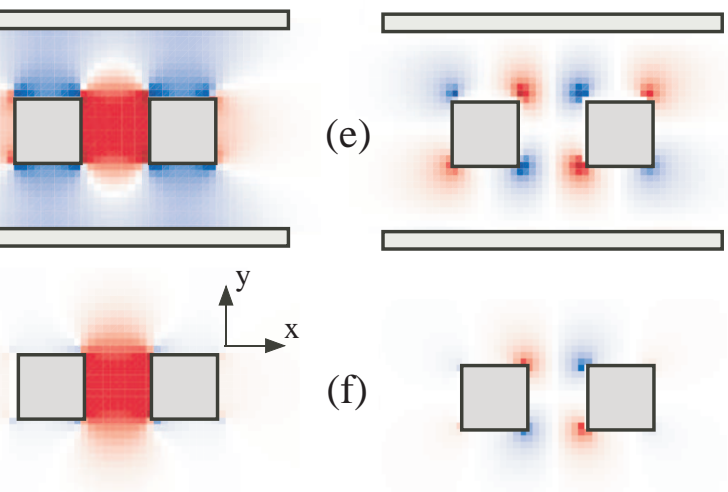

$\Delta \mathrm{T}_{\mathrm{xx}}$ (f)

(e)

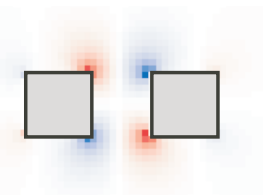

$\Delta \mathrm{T}_{\mathrm{xy}}$
FIG. 8: (Color) (a-f): TM stress map of the geometry in Fig. 7 for various $h$. The interaction stress tensors $\left\langle T_{x x}\right\rangle$ (left) and $\left\langle T_{x y}\right\rangle$ (right) for: (a),(d): $h=0.5 a$; (b),(e): $h=a$; and (c),(f): $h=2 a$, where blue/white/red $=$ repulsive/zero/attractive.

To further explore the source of the $h$-dependence, we plot the TM interaction-stress maps $\Delta\left\langle T_{x x}\right\rangle$ and $\Delta\left\langle T_{x y}\right\rangle$ in Fig. 8, for the perfect-metal squares at a typical frequency $w=2 \pi c / a$, and for varying distances from the metal plates $(h=0.5,1.0,2.0)$. As shown, the magnitudes of both the $x x(\mathrm{a}-\mathrm{c})$ and $x y(\mathrm{~d}-\mathrm{f})$ components of the stress tensor change dramatically as the metal plates are brought closer to the squares. For example, one change in the force integral comes from $T_{x y}$, which for isolated squares has an asymmetric pattern at the four corners that will contribute to the attractive force, whereas the presence of the plates induces a more symmetric pattern of stresses at the four corners that will have nearly zero integral. This results in a decreasing TM force with decreasing $h$ as observed in Fig. 7. Because stress maps indicate where bodies interact and with what signs, it may be useful in future work to explore whether they can be used to design unusual behaviors such as non-additive, non-monotonic, or even repulsive forces.

\section{Two-dimensional dielectric pistons}

Our method is also capable, without modification, of handling arbitrary dielectric materials. The calculation of general dispersive media can be performed with minor or no additional computational effort, since the computations at different $w$ are independent. Unlike most previously published techniques, which do not easily generalize to non-perfect metals, the stress tensor approach does not distinguish between the two regimes, and computational methods for inhomogeneous dielectric materials are widely available. Furthermore, we reiterate that along the imaginary- $\omega$ axis, $\varepsilon$ is purely real and positive even for dissipative materials (which have complex $\varepsilon$ on the real- $\omega$ axis), greatly simplifying computations.

The method's ability to handle dielectric structures is demonstrated below, where the Casimir force between the two squares is shown for two different cases:

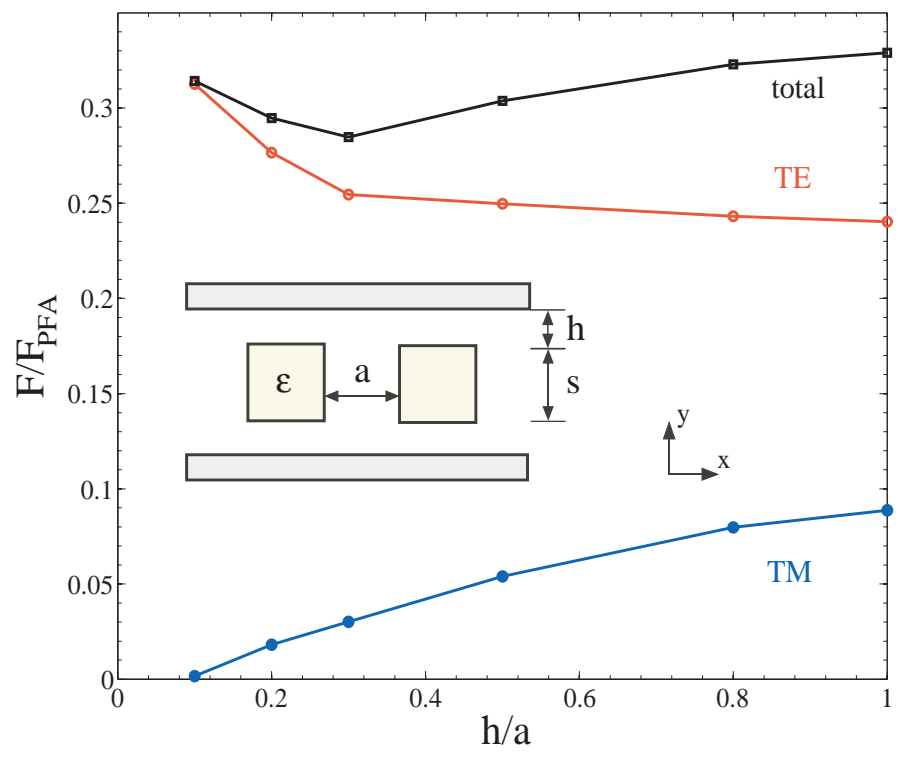

FIG. 9: (Color) Casimir force between 2d ( $z$-invariant fields) dielectric $(\varepsilon=4)$ squares $F / F_{\mathrm{PFA}}$, vs. distance from metal plate $h$ (inset), normalized by the total force (TE+TM) obtained using the PFA (Here, the PFA force is computed for $x$-infinite slabs of dielectric $\varepsilon=4)$. The total force is plotted (black squares) along with the TE (red dots) and TM (blue circles) contributions.

First, we compute the force between two squares made of dielectric material with $\varepsilon=4$ (an artificial mathematical choice for illustration purposes), whereas the parallel plates are still perfect metal. The result is shown on the plot of Fig. 9. As might be expected, the dielectric squares have a weaker interaction than the perfect-metal squares, but are still non-monotonic. 


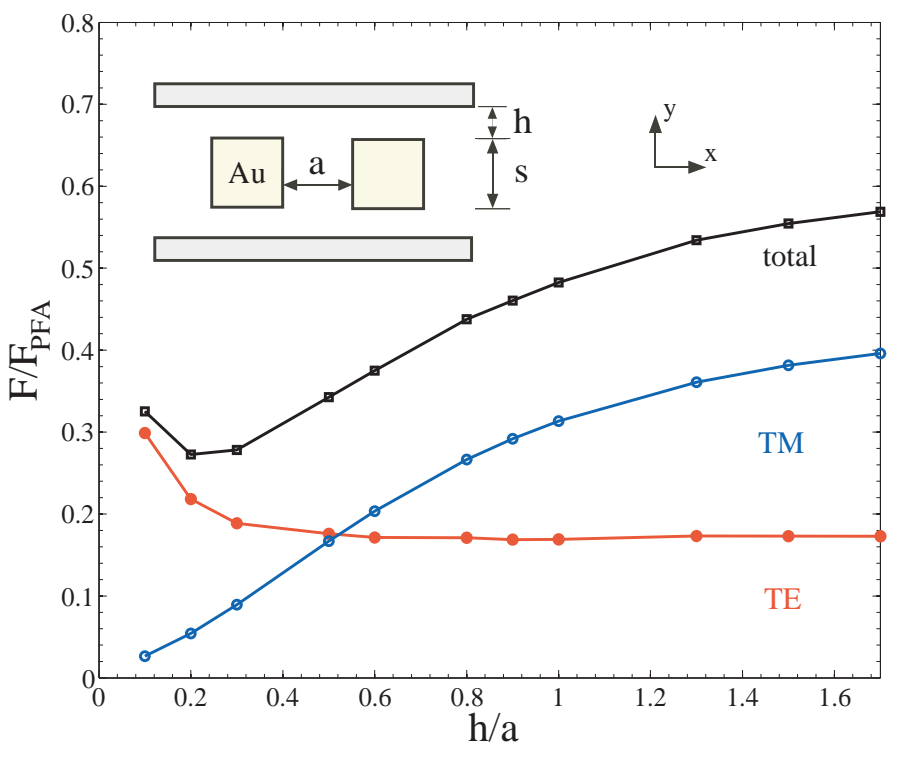

FIG. 10: (Color) Casimir force between 2d ( $z$-invariant fields) gold squares $F / F_{\mathrm{PFA}}$, vs. distance from metal plate $h$ (inset), normalized by the total force (TE+TM) obtained using the PFA. (Here, the PFA force is computed for $x$-infinite gold slabs). The total force is plotted (black squares) along with the TE (red dots) and TM (blue circles) contributions.

Second, as a more interesting example, the squares are made of gold with a Drude dispersion taken from experiment, again with adjacent perfect metallic plates. In particular, the following Drude model is used for the material dispersion of gold [87]:

$$
\varepsilon(\omega)=1-\frac{\omega_{p}^{2}}{\omega\left(\omega+i \Gamma_{p}\right)}
$$

with $\omega_{p}=1.37 \times 10^{16} \mathrm{~Hz}$ and $\Gamma_{p}=5.32 \times 10^{13} \mathrm{~Hz}$, corresponding to $\omega_{p}=7.2731$ and $\Gamma_{p}=0.028243$ in our units of $2 \pi c / a$, for $a=1 \mu \mathrm{m}$. For $\omega=i w$, this is real and positive, as expected. The resulting force is shown in Fig. 10. Not surprisingly, the gold squares have a weaker interaction than perfect-metal squares, since at large $w=\operatorname{Im} \omega$ the dielectric constant $\varepsilon$ goes to 1 .

\section{E. Three-dimensional piston}

The previous $2 \mathrm{~d}$ calculations are important in at least two ways: first, they allow us to check the stress tensor method against previous piston calculations in the $h \rightarrow 0$ limit, while exploring an interesting new geometry; second, based on their results, one might predict a similar behavior for the force per unit length in the analogous $3 \mathrm{~d}$ $z$-invariant geometry, shown in the inset of Fig. 11. Indeed, this is the case: the non-monotonic force in three dimensions is shown in Fig. 11. As discussed in Sec. VI, the integrand for a $z$-invariant perfect-metallic structure differs from the two-dimensional integrand only by a fac- tor of $\pi w$, and therefore the $3 \mathrm{~d}$ force is obtained from the $2 \mathrm{~d}$ calculations with very little additional computation.

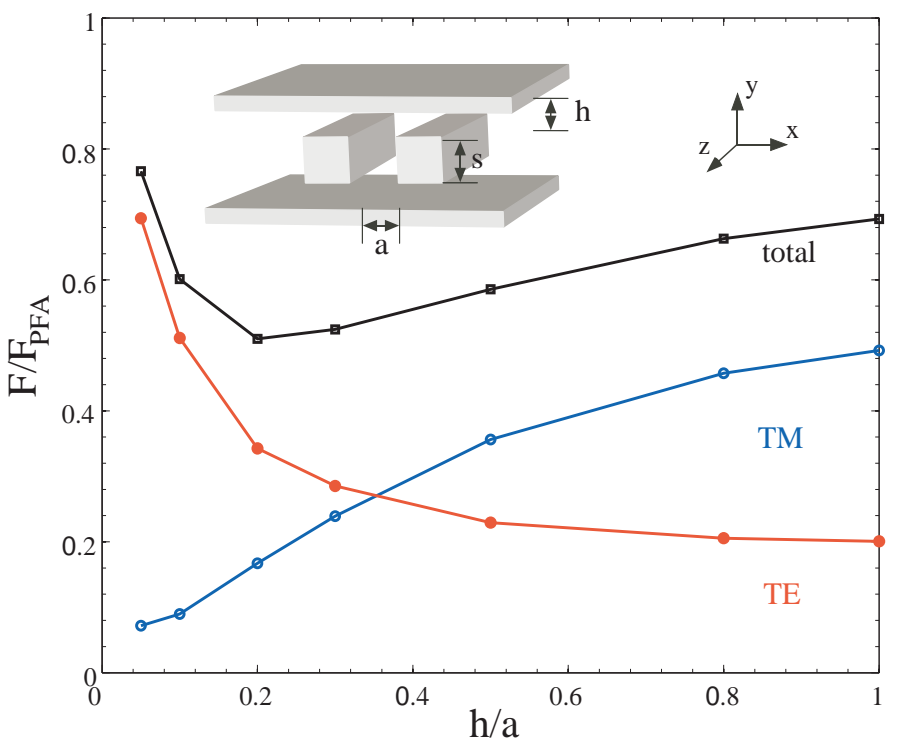

FIG. 11: (Color) Casimir force per unit length between $z$ invariant $3 \mathrm{~d}$ metal blocks $F / F_{\mathrm{PFA}}$, vs. distance from metal plate $h$ (inset), normalized by the total force (TE+TM) obtained using the PFA, $F_{\mathrm{PFA}}=\hbar c s \pi^{2} / 480 a^{4}$. The total force is plotted (black squares) along with the TE (red dots) and TM (blue circles) contributions.

Again, in the $h=0$ limit there are known analytical solutions for this geometry based on the ray-optics method 88] or the zeta-function technique 89. Linearly extrapolating our plot to $h=0$, we reproduce these results to within $\approx 2-3 \%$.

\section{BEYOND FINITE-DIFFERENCE METHODS}

Above, we implemented the stress-tensor integration using a finite-difference frequency-domain approach to compute the Green's function. While sufficient for a proof-of-concept implementation, one would like to use more sophisticated methods in order to explore complex geometries more quickly, especially in three dimensions. The primary drawbacks of the finite-difference scheme are threefold. First, the material discontinuities imply that the error converges only linearly with resolution, although there are techniques to improve this to quadratic convergence 82 . Second, non-uniform resolution would be desirable to handle small features, such as the narrow channels in the piston structure for small $h$. Third, the stress-tensor integrand is a discretized, non-smooth function of space, meaning that we must evaluate it at a number of grid points proportional to the resolution (or resolution squared, in three dimensions); in general, the integrand must therefore be evaluated $O\left(N^{(d-1) / d}\right)$ times for a $d-1$ dimensional surface in $d$ dimensions, 
leading to at best $O\left(N^{2-1 / d}\right)$ complexity. To address these drawbacks, we consider two standard approaches to solving partial differential equations in a more efficient manner in complex geometries: finite-element and boundary-element methods.

Finite-element methods can employ a non-uniform volume discretization, via an unstructured mesh, that can both put more resolution where it is needed and conform to the material interfaces to obtain higher-order accuracy 60, 61, 62, 63. However, from the perspective of Casimir-force calculations, finite-element methods seem to have two potential drawbacks. First, because space is still discretized, the stress tensor is again not a smooth function of space and its accurate integration requires that the Green's function be evaluated at many mesh points. Second, there may be a problem with regularization: although the Green's function does not diverge in discretized space, with a non-uniform resolution this effective regularization varies at different points. Unless there is a way to locally regularize the problem (subtracting a vacuum Green's function computed at the local spatial resolution), this may lead to unphysical, nonconvergent forces.

Boundary-element methods (BEMs) involve a discretization in terms of unknowns only at the interfaces between different materials - these surface unknowns are coupled to one another via the (known) Green's functions of the homogeneous regions [60, 61, 62, 65, 66, 67. They are thus ideal for open problems, in which the bodies are surrounded by infinite volumes of empty space, since those infinite regions are treated analytically. Using the fast-multipole method (FMM) or other fast integralequation methods 60, 61, 62, 65, BEMs can solve for the surface unknowns, and hence the Green's function, in $O(N \log N)$ time for $N$ discretized unknowns, multiplied by a number of iterations $(\ll N)$ that depends on the condition number of the matrix and the preconditioning 69]. Furthermore, BEMs have two unique advantages when applied to the problem of Casimir forces. First, the regularization can be performed analytically: since BEMs express the Green's function as the sum of the vacuum Green's function plus a set of contributions from surface currents, the vacuum Green's function can be trivially subtracted analytically. Second, because space is not discretized, the stress tensor in a BEM will be a smooth (infinitely differentiable) function of spacethis means that the spatial integral can be performed with exponentially-convergent smooth quadrature methods. Therefore, the number of times that the Green's function must be computed is determined only by the convergence of the smooth multidimensional quadrature in $2+1$ dimensions (space + frequency), independent of $N$.

For these reasons, we suspect that BEMs will lead to the most efficient methods to compute Casimir forces for complicated structures in three dimensions. Moreover, all that needs to be done is to take an existing BEM Green's function solver and change it to solve for the imaginary- $\omega$ Green's function. Because the imaginary- $\omega$ Green's functions are exponentially decaying (and approach the familiar Poisson kernel as $\omega \rightarrow 0$ ), such a fast solver should actually be simpler than the corresponding real- $\omega$ solver, nor are the singularities in the Green's function any worse. And, as mentioned previously, the resulting matrix equation is real-symmetric and positivedefinite for imaginary $\omega$, unlike the real- $\omega$ case, even for dissipative materials. In short, there do not appear to be any substantial unsolved algorithmic problems involved in implementing a BEM for Casimir forces.

\section{COMPARISON TO OTHER METHODS}

In this section, we briefly compare the stress-tensor approach with other known exact numerical methods applicable to arbitrary geometries, focusing mostly on the computational aspects. In particular, we examine the methods of Emig et al. 59. and Gies et al. 58.

Emig's method, applicable to both separable and nonseparable geometries and not limited to perfect metallic structures (although currently only demonstrated in perfect metallic separable geometries), involves a surface parameterization of the Green's function. Specifically, the Casimir energy is given in terms of an integral over imaginary frequencies of the change in the photon density of states (DOS) $\delta \rho(i w)$ :

$$
U=\int \frac{\hbar w}{2} \delta \rho(i w) d w
$$

similar to the expression in Eq. (4). However, the crucial aspect of this method lies in the evaluation of the DOS, given by [59]:

$$
\delta \rho(i w)=\frac{1}{\pi} \frac{\partial}{\partial w} \operatorname{tr} \ln \left(M_{\infty}^{-1} M\right) .
$$

Here $M$ is an $N \times N$ dense matrix, where $N$ is the number of surface degrees of freedom, whose entries are in terms of the imaginary- $\omega$ vacuum Green's function $\mathbf{G}\left(i w ; \mathbf{x}, \mathbf{x}^{\prime}\right)$ evaluated on the surface of each body. $M_{\infty}$ is the same matrix for the case where the bodies are infinitely far apart. Thus, the trace in Eq. 18 is analogous to an integration over the surfaces of all the bodies. Inverting a dense matrix, multiplying two dense matrices, and taking the log of a dense matrix all require $O\left(N^{3}\right)$ time (for practical algorithms) and $O\left(N^{2}\right)$ storage [90.

We should comment however, that Emig's method is closely related to a boundary-element method (BEM) as discussed in the previous section. BEMs also involve parameterization in terms of surface degrees of freedom, which are also coupled in terms of vacuum Green's functions, leading to a dense matrix which must be inverted to compute the inhomogeneous Green's function. By recognizing this relationship, one should be able to exploit fastmultipole and similar $O(N \log N)$ techniques to accelerate Emig's method. In particular, computations such as 
$M_{\infty}^{-1} M$ involve the solution of $N$ linear equations, each of which can employ iterative methods with $O(N)$ storage, and similar iterative methods may also be available for computing matrix logarithms 90 . At best, this leads to $O\left(N^{2} \log N\right)$ time. However, this is still much less efficient than the stress-tensor BEM approach discussed in the previous section, because the latter requires only $\ll N$ linear equations to be solved (the number of linear equations to be solved is determined by a smooth spatial quadrature, independent of $N$ ).

A second exact computational method available is that of Ref. 58, based on a "worldline" approach. In this method, the Casimir energy is represented via a scalar field in a smooth background potential, and the effective action is obtained via a Feynman path integral over all proper time worldlines using a Monte-Carlo approximation. The method has only been formulated for the TM polarization with perfect-metal bodies, although preliminary generalizations have been suggested [91. Specifically, the method expresses the Casimir energy between two bodies as the integral of a functional $\Theta_{\Sigma}$ over closed paths $\mathbf{x}(\tau)$ of length (proper time) $T$ and center of mass y:

$$
U=-\frac{1}{8 \pi^{2}} \int_{0}^{\infty} \frac{d T}{T^{3}} \int d^{3} \mathbf{y}\left\langle\Theta_{\Sigma}[\mathbf{x}(\tau)]\right\rangle_{\mathbf{x}}
$$

where $\Theta_{\Sigma}[\mathbf{x}(\tau)]$ is a worldline functional (similar to a step function) defined to be $\Theta_{\Sigma}[\mathbf{x}(\tau)]=1$ if the path $\mathbf{x}(\tau)$ intersects a surface and 0 otherwise 91]. This integration is performed by generating an ensemble of $n_{L}$ random $N$-point paths $\mathbf{x}(\tau)$ and evaluating $\Theta_{\Sigma}$ for each one. The computational complexity $O\left(n_{L} \cdot N \cdot \# \mathbf{y} \cdot \# T\right)$ (where $\# \mathbf{y}$ and $\# T$ are the number of $\mathbf{y}$ and $T$ integrand evaluations, respectively), therefore, depends on quantities such as the precise statistical rate of convergence of this integral (error $\sim 1 / \sqrt{n_{L}}$ ), which in turn depends on the geometry and on the manner in which the path ensembles are generated and integrated. Ref. 58 does not present a general analysis of these quantities, nor will we do so here. However, in the specific case of the force between a radius- $R$ sphere and a plate separated by a distance $a$, Ref. 58 shows that $N \gg a^{2} / R^{2}$ for large $a / R$. This does not include the $O\left(n_{L} \cdot \# \mathbf{y} \cdot \# T\right)$ factors, although it seems likely that $\# \mathbf{y}$ is at least $\sim N$ (so that the spatial resolutions are comparable), in which case the time scaling would be at least $a^{4} / R^{4} \sim N^{2}$. In comparison, a BEM for the same geometry (exploiting the cylindrical symmetry to reduce it to a $2 \mathrm{~d}$ problem similar to Ref. 58) should require degrees of freedom $N$ that never scale worse than linearly with the relevant lengthscale, and time that scales with $N \log N$ rather than $N^{2}$. A general comparison seems difficult, however, and a detailed statistical study is outside the scope of this paper.

\section{CONCLUDING REMARKS}

The general considerations involved in designing a purely computational method are often quite different from those involved in designing an analytical method. For this reason, we believe it is most fruitful to start back at the earliest possible formulations and proceed using the new computational perspective, rather than attempting to add more and more corrections to analytical methods for specific geometries. Moreover, since decades of research have gone into the development of numerical methods for classical electromagnetism, culminating in methods applicable to complicated inhomogeneous threedimensional geometries, it is desirable to seek approaches for the Casimir force that exploit these developments. We believe that the stress-tensor approach, developed for analytical calculations several decades ago, provides the ideal formulation for a computational approach exploiting standard numerical techniques.

In the future, we would like to employ the stresstensor approach to study Casimir forces and torques in more realistic and/or more unusual structures. The large and growing number of interesting applications of the Casimir effect and the ongoing experimental work on non-standard geometries [8] provide an environment in which the generality and strengths of the stress-tensor method could be exploited. In addition, we are currently implementing more efficient boundary-element versions of our approach in three dimensions.

We would also like to investigate related computational problems. One immediate possibility is to compute the net torque on a body, instead of the net force. Classically, given the stress tensor $\mathbf{T}$, one can compute the torque by integrating $\mathbf{r} \times(\mathbf{T} \cdot d \mathbf{S})$ [92]. This has been exploited by several authors to compute classical electromagnetic torques 93, 94, 95. Similarly, the Casimir torque can be obtained by using the mean $\langle\mathbf{T}\rangle$ from the fluctuation-dissipation theorem, just as for the net force [34]. Therefore, one can compute torques by almost the same method as above, via repeated evaluations of the classical Green's function. Several authors have computed Casimir torques in parallel-plate and perfectmetallic wedge geometries [96, 97, 98, 99, 100; other interesting structures include the Casimir "pendulum" 553 as well as corrugated surfaces [101, although these two structures have only been evaluated by methods with uncontrolled approximations.

\section{Acknowledgements}

This work was supported in part by the Nanoscale Science and Engineering Center (NSEC) under NSF contract PHY-0117795 and by a Department of Energy (DOE) Computational Science Graduate Fellowship under grant DE-FG02-97ER25308. D. I. gratefully acknowledges support from the Netherlands Organisation for Scientific Research (NWO), under the IRI Scheme 
Vernieuwingsimpuls VIDI-680-47-209. We are also grateful to M. Povinelli, Federico Capasso, R. L. Jaffe, and M. Kardar for inspiration and helpful discussions.

\section{Appendix}

In what follows, we derive the force per unit length in translation-invariant structures in terms of an integral over the solutions of Bloch-periodic problems. This is not a new idea, but an explicit general derivation starting from the spatial integral of the stress tensor, including the case of finite periodicity, seems difficult to find in the literature. As given above by Eq. (13), the force per unit length can be written as:

$$
\frac{\mathbf{F}}{L}=\frac{1}{L} \int_{0}^{\infty} d w \sum_{n=0}^{N-1} \iint \mathbf{T}(i w ; \mathbf{r}-n \Lambda \hat{\mathbf{z}}) \cdot d \mathbf{S}
$$

for a periodic structure in the $z$-direction with period $\Lambda$, and size $L=N \Lambda$ with periodic boundary conditions (ultimately, we will take the limit as $N \rightarrow \infty$ ). As in Eq. (11), the stress tensor is expressed via the Green's function, and therefore Eq. 20 can be decomposed into individual terms of the form:

$$
\frac{1}{L} \int_{0}^{\infty} d w \sum_{n=0}^{N-1} \iint \mathbf{G}\left(i w ; \mathbf{r}_{n}, \mathbf{r}_{n}\right) d A
$$

where $\mathbf{r}_{n}=\mathbf{r}-n \Lambda \hat{\mathbf{z}}$ and the Green's function is given as in Eq. (7) by the solution of:

$$
\mathbf{G}_{k}\left(i w ; \mathbf{x}, \mathbf{x}^{\prime}\right)=\hat{O}^{-1} \delta^{3}\left(\mathbf{x}-\mathbf{x}^{\prime}\right) \hat{\mathbf{e}}_{k},
$$

in which $\hat{O}$ denotes the linear operator $\hat{O}=$ $\left(\nabla \times \nabla \times+w^{2} \varepsilon\right)$. In the following, we will focus on the periodic $z$ direction and leave the $x$ and $y$ coordinates implicit for simplicity. That is, we will write e.g. $\delta\left(z-n \Lambda-z^{\prime}\right)$ instead of $\delta\left(\mathbf{r}_{n}-\mathbf{r}^{\prime}\right)$.

At this point, we can re-express the delta function over the periodic direction $z$ in terms of the Fourier identity:

$\delta\left(z-z^{\prime}-n \Lambda\right)=\frac{1}{N} \sum_{\ell=0}^{N-1} \sum_{m=0}^{N-1} \delta\left(z-z^{\prime}-\ell \Lambda-n \Lambda\right) e^{\frac{2 \pi i}{N} \ell m}$
Substituting this into Eq. 21, we will move the $\sum_{m}$ outside the $\sum_{n}$ and consider the action of $\hat{O}^{-1}$ on the remaining summation:

$$
J_{n, m}=\sum_{\ell=0}^{N-1} \delta\left(z-z^{\prime}-\ell \Lambda-n \Lambda\right) e^{\frac{2 \pi i}{N} \ell m}=J_{0, m} e^{-\frac{2 \pi i}{N} m n}
$$

where we have used the periodic boundary conditions in $L$ to realize that $J_{n, m}$ is a cyclic shift of $J_{0, m}$ with a phase factor. Now, we must operate $\hat{O}^{-1}$ on $J_{0, m} \hat{\mathbf{e}}_{k}$ and evaluate at $z^{\prime}=z-n \Lambda$. However, this corresponds to finding the field from a Bloch-periodic current source, and such a field is also Bloch-periodic. Therefore:

$$
\left.\left(\hat{O}^{-1} J_{0, m} \hat{\mathbf{e}}_{k}\right)\right|_{z^{\prime}=z-n \Lambda}=\left.\left(\hat{O}^{-1} J_{0, m} \hat{\mathbf{e}}_{k}\right)\right|_{z^{\prime}=z} e^{\frac{2 \pi i}{N} m n},
$$

At this point, we have completely eliminated the $n$ dependence from the evaluation of the Green's function $\hat{O}^{-1} J_{0, m} \hat{\mathbf{e}}_{k}$, and the phase factors from Eq. (24) and Eq. 25 cancel. The remaining summation $\sum_{n}$ simply yields $N$, which cancels the $1 / N$ factor from Eq. 23. Equation (21) therefore becomes:

$$
\frac{1}{L} \int_{0}^{\infty} d w \sum_{m=0}^{N-1} \iint \mathbf{G}\left(i w, m ; \mathbf{r}_{n}, \mathbf{r}_{n}\right) d A
$$

where $\mathbf{G}_{k}(i w, m)=\hat{O}^{-1} J_{0, m} \hat{\mathbf{e}}_{k}$, the field from a Blochperiodic sum of delta-function sources. Finally, we can now take the limit $N \rightarrow \infty$ by turning $\sum_{m}$ into an integral:

$$
\lim _{N \rightarrow \infty} \frac{1}{L} \sum_{m=0}^{N-1}=\frac{1}{2 \pi \Lambda} \int_{-\pi / \Lambda}^{\pi / \Lambda} d k_{z}
$$

where $k_{z}$ is the Bloch wavevector $\left(k_{z}=2 \pi m / N\right)$. We therefore obtain Eq. (15).
[1] H. B. G. Casimir, "On the attraction between two perfectly conducting plates," Proc. K. Ned. Akad. Wet., vol. 51, pp. 793-795, 1948.

[2] P. W. Milonni, The Quantum Vacuum: An Introduction to Quantum Electrodynamics. San Diego: Academic Press, 1993.

[3] E. M. Lifshitz and L. P. Pitaevskii, Statistical Physics: Part 2. Oxford: Pergamon, 1980.

[4] G. Plunien, B. Muller, and W. Greiner, "The Casimir effect," Phys. Rep., vol. 134, no. 87, 1986.

[5] V. M. Mostepanenko and N. N. Trunov, The Casimir Effect and its Applications. Oxford: Clarendon Press, 1997.

[6] S. K. Lamoreaux, "Demonstration of the Casimir force in the 0.6 to $6 \mu \mathrm{m}$ range," Phys. Rev. Lett., vol. 78, p. 5, 1997.

[7] R. Onofrio, "Casimir forces and non-Newtonian gravitation," New J. Phys., vol. 8, p. 237, 2006. 
[8] F. Capasso, J. N. Munday, D. Iannuzzi, and H. B. Chan, "Casimir forces and quantum electrodynamical torques: Physics and nanomechanics," IEEE J. Selected Topics in Quant. Elec., vol. 13, no. 2, pp. 400-415, 2007.

[9] G. Bressi, G. Carugno, R. Onofrio, and G. Ruoso, "Measurement of the Casimir force between parallel metallic surfaces," Phys. Rev. Lett., vol. 88, p. 041804, 2002.

[10] J. Mahanty and B. W. Ninham, Dispersion Forces. London: Academic Press, 1976.

[11] J. N. Israelachvili, Intermolecular and Surface Forces. London: Academic Press, 1991.

[12] P. H. G. M. van Blokland and J. T. G. Overbeek, "Van der Waals forces between objects covered with a chromium layer," J. Chem. Soc. Far. Trans. I, vol. 74, pp. 2637-2651, 1978.

[13] S. K. Lamoreaux, "Demonstration of the Casimir force in the 0.6 to $6 \mu \mathrm{m}$ range," Phys. Rev. Lett., vol. 78, pp. 5-8, 1997.

[14] U. Mohideen and A. Roy, "Precision measurement of the Casimir force from 0.1 to $0.9 \mu \mathrm{m}$," Phys. Rev. Lett., vol. 81, p. 4549, 1998.

[15] A. Roy, C. Y. Lin, and U. Mohideen, "Improved precision measurement of the Casimir force," Phys. Rev. D, vol. 60, p. 111101(R), 1999.

[16] B. W. Harris, F. Chen, and M. U., "Precision measurement of the Casimir force using gold surfaces," Phys. Rev. A, vol. 62 , p. 052109,2000

[17] H. B. Chan, V. A. Aksyuk, R. N. Kleinman, D. J. Bishop, and F. Capasso, "Quantum mechanical actuation of microelectromechanical systems by the Casimir force," Science, vol. 291, pp. 1941-1944, 2001.

[18] H. B. Chan, V. A. Aksyuk, R. N. Kleinman, D. J. Bishop, and F. Capasso, "Nonlinear micromechanical Casimir oscillator," Phys. Rev. Lett., vol. 87, p. 211801, 2001.

[19] R. S. Decca, D. Lopez, E. Fischbach, and D. E. Krause, "Measurement of the Casimir force between dissimilar metals," Phys. Rev. Lett., vol. 91, p. 050402, 2003.

[20] R. S. Decca, F. Fischbach, G. L. Klimchitskaya, D. E. Krause, D. Lopez, and V. M. Mostepanenko, "Improved tests of extra-dimensional physics and thermal quantum field theory from new Casimir force measurements," Phys. Rev. D, vol. 68, p. 116003, 2003.

[21] D. Iannuzzi, M. Lisanti, and F. Capasso, "Effect of hydrogen-switchable mirrors on the Casimir force," Proc. Nat. Ac. Sci. USA, vol. 101, pp. 4019-4023, 2004.

[22] M. Lisanti, D. Iannuzzi, and F. Capasso, "Observation of the skin-depth effect on the Casimir force between metallic surfaces," Proc. Nat. Ac. Sci. USA, vol. 102, pp. 11989-11992, 2005.

[23] T. Ederth, "Template-stripped gold surfaces with 0.4$\mathrm{nm}$ rms roughness suitable for force measurements: Application to the Casimir force in the 20-100-nm range," Phys. Rev. A, vol. 62, p. 062104, 2000.

[24] O. Kenneth and I. Klich, "Opposites attract: A theorem about the Casimir force," Phys. Rev. Lett., vol. 97, p. 160401, 2006.

[25] T. H. Boyer, "Van der Waals forces and zero-point energy for dielectric and permeable materials," Phys. Rev. A, vol. 9, pp. 2078-2084, 1974.

[26] V. Hushwater, "Repulsive Casimir force as a result of vacuum radiation pressure," Am. J. Phys., vol. 65, no. 5, pp. 381-384, 1996.

[27] O. Kenneth, I. Klich, A. Mann, and M. Revzen, "Re- pulsive Casimir forces," Phys. Rev. Lett., vol. 89, no. 3 p. 033001, 2002.

[28] D. Iannuzzi and F. Capasso, "Comment on "repulsive Casimir forces"," Phys. Rev. Lett., vol. 91, no. 2, p. 029101, 2003.

[29] C.-G. Shao, D.-L. Zheng, and J. Luo, "Repulsive Casimir effect between anisotropic dielectric and permeable plates," Phys. Rev. A, vol. 74, p. 012103, 2006.

[30] F. Intravaia and A. Lambrecht, "Surface plasmon modes and the Casimir energy," Phys. Rev. Lett., vol. 94, p. 110404, 2005.

[31] Y. Sherkunov, "Van der Waals interaction of excited media," Phys. Rev. A, vol. 72, p. 052703, 2005.

[32] J. N. Fuchs, A. Recati, and W. Zwerger, "Oscillating Casimir force between impurities and one-dimensional Fermi liquids," Phys. Rev. A, vol. 75, p. 043615, 2007.

[33] K. W. Wetz, "Repulsive Casimir force between dielectric planes," arXiv:quant-ph/0109134, 2001.

[34] E. M. Lifshitz Sov. Phys. JETP, vol. 2, p. 73, 1956.

[35] I. E. Dzyaloshinskiü, E. M. Lifshitz, and L. P. Pitaevskiŭ, "The general theory of van der Waals forces," $A d v$. Phys., vol. 10, no. 38, pp. 165-209, 1961.

[36] M. S. Tomaš, "Casimir force in absorbing multilayers," Phys. Rev. A, vol. 66, p. 052103, 2002.

[37] M. Bordag, U. Mohideen, and V. M. Mostepanenko, "New developments in the Casimir effect," Phys. Rep., vol. 353, pp. 1-205, 2001.

[38] T. Emig, A. Hanke, R. Golestanian, and M. Kardar, "Probing the strong boundary shape dependence of the Casimir force," Phys. Rev. Lett., vol. 87, p. 260402, 2001.

[39] C. Genet, A. Lambrecht, P. Maia Neto, and S. Reynaud, "The Casimir force between rough metallic plates," $E u$ rophys. Lett., vol. 62, p. 484, 2003.

[40] T. Emig, A. Hanke, R. Golestanian, and M. Kardar, "Normal and lateral Casimir forces between deformed plates," Phys. Rev. A, vol. 67, p. 022114, 2003.

[41] T. Emig, "Casimir forces: An exact approach for periodically deformed objects," Europhys. Lett., vol. 62, p. 466, 2003.

[42] H. Gies and K. Klingmuller, "Casimir effect for curved geometries: Proximity-force-approximation validity limits," Phys. Rev. Lett., vol. 96, p. 220401, 2006.

[43] P. A. Maia Neto, A. Lambrecht, and S. Reynaud, "Roughness correction to the Casimir force: Beyond the proximity force approximation," Europhys. Lett., vol. 69 , p. $924,2005$.

[44] A. Rodriguez, M. Ibannescu, D. Iannuzzi, F. Capasso, J. D. Joannopoulos, and S. G. Johnson, "Computation and visualization of Casimir forces in arbitrary geometries: Non-monotonic lateral-wall forces and failure of proximity force approximations," Phys. Rev. Lett, In Print, 2007.

[45] M. Tajmar, "Finite element simulation of Casimir forces in arbitrary geometries," Intl. J. Mod. Phys. C, vol. 15, no. 10, pp. 1387-1395, 2004.

[46] R. Sedmik, I. Vasiljevich, and M. Tajmar, "Detailed parametric study of Casimir forces in the Casimir Polder approximation for nontrivial 3d geometries," $J$. Computer-Aided Mat. Des., vol. 14, no. 1, pp. 119-132, 2006.

[47] M. Schaden and L. Spruch, "Infinity-free semiclassical evaluation of Casimir effects," Phys. Rev. A, vol. 58, pp. 935-953, 1998. 
[48] R. Balian and B. Duplantier, "Electromagnetic waves near perfect conductors. II. Casimir effect," Ann. Phys., vol. 112, pp. 165-208, 1978.

[49] A. Lambrecht, P. A. Maia Neto, and S. Reynaud, "The Casimir effect within scattering theory," New Journal of Physics, vol. 8, no. 243, 2006.

[50] R. L. Jaffe and A. Scardicchio, "Casimir effect and geometric optics," Phys. Rev. Lett., vol. 92, p. 070402, 2004.

[51] M. Bordag, "Casimir effect for a sphere and a cylinder in front of a plane and corrections to the proximity force theorem," Phys. Rev. D, vol. 73, p. 125018, 2006.

[52] T. Emig, A. Hanke, R. Golestanian, and M. Kardar, "Probing the strong boundary shape dependence of the Casimir force," Phys. Rev. Lett., vol. 87, p. 260402, 2001.

[53] A. Scardicchio and R. L. Jaffe, "Casimir effects: An optical approach I. foundations and examples," arXiv.org preprint archive, pp. quant-ph/0406041, 2004.

[54] I. Brevik, E. K. Dahl, and G. O. Myhr, "Casimir force on a micrometer sphere in a dip: proposal of an experiment," J. Phys. A: Mathematical and General, vol. 38, pp. 49-56, 2005.

[55] R. L. Jaffe, S. G. Johnson, A. Rodriguez, and S. Zaheer unpublished results, 2007.

[56] R. Büscher and T. Emig, "Geometry and spectrum of Casimir forces," Phys. Rev. Lett., vol. 94, p. 133901, 2005.

[57] T. Emig, R. L. Jaffe, M. Kardar, and A. Scardicchio, "Casimir interaction between a plate and a cylinder," Phys. Rev. Lett., vol. 96, p. 080403, 2006.

[58] H. Gies, K. Langfeld, and L. Moyaerts, "Casimir effect on the worldline," J. High Energy Phys., no. 06, p. 018, 2003.

[59] T. Emig and R. Büscher, "Towards a theory of molecular forces between deformed media," Nucl. Phys. B, vol. 696, p. 468, 2004.

[60] W. C. Chew, J. Jian-Ming, E. Michielssen, and S. Jiming, Fast and Efficient Algorithms in Computational Electromagnetics. Norwood, MA: Artech, 2001.

[61] J. L. Volakis, A. Chatterjee, and L. C. Kempel, Finite Element Method Electromagnetics: Antennas, Microwave Circuits, and Scattering Applications. New York: IEEE Press, 2001.

[62] J. Jin, The Finite Element Method in Electromagnetics. New York: Wiley, second ed., 2002.

[63] Y. Zhu and A. C. Cangellaris, Multigrid Finite Element Methods for Electromagnetic Field Modelling. Hooboke, NJ: John Wiley and Sons, 2006.

[64] J. P. Boyd, Chebychev and Fourier Spectral Methods. New York: Dover, 2nd ed., 2001.

[65] W. Hackbush and B. Verlag, Integral Equations: Theory and Numerical Treatment. Basel, Switzerland: Birkhauser Verlag, 1995.

[66] W. C. Chew, J. M. Jin, E. Michelssen, and J. M. Song, "Fast solution methods in electromagnetics," IEEE Trans. Ant. Prop., vol. 45, no. 3, pp. 533-543, 1997.

[67] S. M. Rao and N. Balakrishnan, "Computational electromagnetics," Current Science, vol. 77, no. 10, pp. 1343-1347, 1999.

[68] A. Christ and H. L. Hartnagel, "Three-dimensional finite-difference method for the analysis of microwavedevice embedding," IEEE Trans. Microwave Theory Tech., vol. 35, no. 8, pp. 688-696, 1987.
[69] B. Zhaojun, J. Demmel, J. Dongarra, A. Ruhe, and H. Van Der Vorst, Templates for the Solution of Algebraic Eigenvalue Problems: A Practical Guide. Philadelphia: SIAM, 2000.

[70] L. P. Pitaevskiı̌, "Comment on "Casimir force acting on magnetodielectric bodies in embedded in media"," Phys. Rev. A, vol. 73, p. 047801, 2006.

[71] K. A. Milton, "The Casimir effect: recent controveries and progress," Journal of Physics A: Mathematical and General, vol. 37, pp. R209-R277, 2004.

[72] F. D. Mazitelli, D. A. Dalvit, and F. C. Lobardo, "Exact zero-point interaction energy between cylinders," New Journal of Physics, vol. 8, no. 240, pp. 1-21, 2006.

[73] S. G. Johnson and J. D. Joannopoulos, "Block-iterative frequency-domain methods for Maxwell's equations in a planewave basis," Opt. Express, vol. 8, no. 3, pp. 173190, 2001.

[74] S. J. Van Enk, "The Casimir effect in dielectrics: A numerical approach," Journal of Modern Optics, vol. 42, no. 2, pp. 321-338, 1995.

[75] L. H. Ford, "Spectrum of the Casimir effect and the Lifshitz theory," Phys. Rev. A, vol. 48, p. 2962, 1993.

[76] J. D. Jackson, Classical Electrodynamics. New York: Wiley, third ed., 1998.

[77] L. D. Landau and E. M. Lifshitz, Quantum Mechanics. Oxford: Butterworth-Heinemann, 3rd ed., 1977.

[78] R. Piessens, E. de Doncker-Kapenga, C. Uberhuber, and D. Hahaner, QUADPACK: A Subroutine Package for Automatic Integration. Berlin: Springer-Verlag, 1983.

[79] U. Trottenberg, C. Ooseterlee, and A. Schüller, Multigrid. London: Academic Press, 2001.

[80] R. Matloob, A. Keshavarz, and D. Sedighi, "Casimir effect for two lossy dispersive dielectric slabs," Phys. Rev. A, vol. 60, no. 5, pp. 3410-3420, 1999.

[81] R. Matloob, "Casimir effect between two conducting plates," Phys. Rev. A, vol. 60, no. 5, pp. 3421-3428, 1999.

[82] A. Farjadpour, D. Roundy, A. Rodriguez, M. Ibanescu, P. Bermel, J. Burr, J. D. Joannopoulos, and S. G. Johnson, "Improving accuracy by subpixel smoothing in the finite-difference time domain," Opt. Lett., vol. 31, pp. 2972-2974, 2006.

[83] J. D. Joannopoulos, R. D. Meade, and J. N. Winn, Photonic Crystals: Molding the Flow of Light. Princeton Univ. Press, 1995.

[84] R. Büscher and T. Emig, "Nonperturbative approach to Casimir interactions in periodic geometries," Phys. Rev. A, vol. 69, p. 062101, 2004.

[85] H. Gies and K. Klingmuller, "Casimir edge effects," Phys. Rev. Lett., vol. 97, p. 220405, 2006.

[86] R. M. Cavalcanti, "Casimir force on a piston," Phys. Rev. D, vol. 69, p. 065015, 2004.

[87] I. Brevik, J. B. Aarseth, J. S. Hoye, and K. A. Milton, "Temperature dependence of the Casimir effect," Phys. Rev. E, vol. 71, no. 056101, 2005.

[88] M. P. Hertzberg, R. L. Jaffe, M. Kardar, and A. Scardicchio, "Attractive Casimir forces in a closed geometry," Phys. Rev. Lett., vol. 95, p. 250402, 2005.

[89] V. M. Marachevsky, "Casimir interaction of two plates inside a cylinder," PRD, vol. 75, p. 085019, 2007.

[90] G. H. Golub and C. F. Van Loan, Matrix Computations. Baltimore, Maryland: The Johns Hopkins University Press, 3rd ed., 1996.

[91] H. Gies and K. Klingmuller, "Worldline algorithms 
for Casimir configurations," Phys. Rev. D, vol. 74, p. 045002, 2006.

[92] L. D. Landau and E. M. Lifshitz, Electrodynamics of Continuous Media. Oxford: Pergamon Press, 1960.

[93] W. L. Collett, C. A. Ventrice, and S. M. Mahajan, "Electromagnetic wave technique to determine radiation torque on micromachines driven by light," Appl. Phys. Lett., vol. 82, no. 16, pp. 2730-2733, 2003.

[94] M. Liu, N. Ji, Z. Lin, and C. S. T., "Radiation torque on a birefringent sphere caused by an electromagnetic wave," Phys. Rev. E, vol. 72, p. 056610, 2005.

[95] M. Popescu, "Prediction of the electromagnetic torque in synchronous machines through Maxwell stress harmonic filter (HFT) method," Electrical Engineering, vol. 89, pp. 117-125, 2006.

[96] S. J. van Enk, "Casimir torque between dielectrics," Phys. Rev. A, vol. 52, no. 4, pp. 2569-2575, 1995.
[97] I. Brevik and M. Lygren, "Casimir effect for a perfectly conducting wedge," Ann. Phys., vol. 51, p. 157, 1996.

[98] C.-G. Shao, A.-H. Tong, and J. Luo, "Casimir torque between birefringent plates," Phys. Rev. A, vol. 72, p. 022102, 2005.

[99] H. Razmi and S. M. Modarresi, "Casimir torque for a perfectly conducting wedge: a canonical quantum field theoretical approach," Int. J. Theor. Phys., vol. 44, no. 2, pp. 229-234, 2005.

[100] J. N. Munday, D. Iannuzzi, Y. Barash, and F. Capasso, "Torque induced on birefringent plates by quantum fluctuations," Phys. Rev. A, vol. 71, p. 042102, 2005.

[101] R. B. Rodrigues, P. A. Maia Neto, A. Lambrecht, and S. Reynaud, "Vacuum-induced torque between corrugated metallic plates," Europhys. Lett., vol. 75, no. 5, pp. 822-828, 2006. 